\title{
Master equation based steady-state cluster perturbation theory
}

\author{
Martin Nuss, "Gerhard Dorn, Antonius Dorda, Wolfgang von der Linden, and Enrico Arrigoni \\ Institute of Theoretical and Computational Physics, Graz University of Technology, 8010 Graz, Austria
}

(Received 12 May 2015; published 16 September 2015)

\begin{abstract}
A simple and efficient approximation scheme to study electronic transport characteristics of strongly correlated nanodevices, molecular junctions, or heterostructures out of equilibrium is provided by steady-state cluster perturbation theory. In this work, we improve the starting point of this perturbative, nonequilibrium Green's function based method. Specifically, we employ an improved unperturbed (so-called reference) state $\hat{\rho}^{S}$, constructed as the steady state of a quantum master equation within the Born-Markov approximation. This resulting hybrid method inherits beneficial aspects of both the quantum master equation as well as the nonequilibrium Green's function technique. We benchmark this scheme on two experimentally relevant systems in the single-electron transistor regime: an electron-electron interaction based quantum diode and a triple quantum dot ring junction, which both feature negative differential conductance. The results of this method improve significantly with respect to the plain quantum master equation treatment at modest additional computational cost.
\end{abstract}

DOI: 10.1103/PhysRevB.92.125128

PACS number(s): 71.15.-m, 71.27.+a, 73.63.Kv

\section{INTRODUCTION}

Electronic transport in the realm of molecular scale junctions and devices has become a subject of intense study in recent years [1-7]. Nowadays, the controlled assembly of structures [8] via electromigration [9-17], the contacting in mechanical break-junction setups [18-21], electronic gating $[17,19,22]$, and measurement via scanning tunneling microscopy [23-26] have become established tools, ultimately opening routes from elementary understanding to device engineering. Prompted by these formidable advances in experimental techniques, the characterization of transport through, e.g., molecules bound by anchor groups to metal electrodes [20,21,27], heterostructures [28,29], or nanostructures on two-dimensional substrates [28,30-35] has become feasible. These constitute the foundation for future applications in electronic devices based on single-electron tunneling [36], quantum interference effects [37-43], spin control [44,45], or even quantum many-body effects $[9,10,12,46,47]$ such as Kondo [48] behavior [49-53].

Typically, the electronic transport through such devices is significantly influenced by electronic correlation effects, which may become large due to the reduced effective dimensionality and/or confined geometries. This is reflected, for instance, in major discrepancies between experimental and theoretical current-voltage characteristics obtained with (uncorrelated) nonequilibrium Green's function [54-57] calculations based on $a b$ initio density functional theory states [1,58-61]. The inclusion of many-body effects in the theoretical description of fermionic systems out of equilibrium [54,62-64] is challenging and an active area of current research [65-73]. Suitable approximations need to be devised in order to solve a finite strongly correlated quantum many-body

\footnotetext{
*martin.nuss@tugraz.at

Published by the American Physical Society under the terms of the Creative Commons Attribution 3.0 License. Further distribution of this work must maintain attribution to the author(s) and the published article's title, journal citation, and DOI.
}

problem out of equilibrium coupled to an infinite environment. Typically, the nonequilibrium setup consists of a correlated central region (system) attached to two leads (environment).

A well-established method for treating such open quantum systems is by means of quantum master equations (ME) [74-79]. Herein, the environment degrees of freedom are integrated out and usually incorporated in a perturbative manner. The ME approach allows a detailed investigation of transport phenomena [44,45] and recent self-consistent extensions attempt to cure some of its long-standing limitations [80].

In the framework of nonequilibrium Green's functions (NEGF), various schemes exist to approximately calculate the electronic self-energy of the correlated region (see, e.g., Refs. [67,81-86]). In cluster approaches, such as cluster perturbation theory (CPT) and its improvement, the variational cluster approach (VCA) [87], the whole system is partitioned into parts which can be treated exactly and determine the self-energy. Originally devised for strongly correlated systems in equilibrium [80,89], both approaches have recently been extended to nonequilibrium situations in the time-dependent case $[90,91]$ as well as in the steady-state $[91,92]$. In previous work we applied the steady-state CPT (stsCPT) to obtain transport characteristics of heterostructures [92], quantum dots [93-95], and molecular junctions [96,97] and obtained good results even in the challenging Kondo regime [48,93,94].

A key issue in the CPT approach is to identify an appropriate many-body state for the disconnected correlated cluster in the central region, as a starting point of perturbation theory, the socalled reference state. Up to now, a common choice in stsCPT is to use an equilibrium state at some temperature $T_{S}$ (often $T_{S}=0$ ) and chemical potential $\mu_{S}$ in-between the values of the leads. Such an ad hoc choice is clearly unsatisfactory. Furthermore, it fails to describe certain quantum interference effects in transport phenomena as, for example, so-called current blocking effects $[44,45,96]$.

The purpose of this work is to improve on stsCPT by constructing a consistent and conceptually more appropriate reference state, given by the steady-state reduced many-body density matrix $\hat{\rho}^{S}$ obtained from a ME in the Born-Markov 
approximation. Within this quantum master equation based stsCPT (ME-CPT), the ambiguity in defining $\mu_{S}$ and $T_{S}$ for the central region is resolved. The equilibrium case, in which $\mu_{S}$ and $T_{S}$ coincide with those of the environment, is automatically included. In contrast to standard ME approaches, lead induced level-broadening effects are accounted for and the noninteracting limit is reproduced exactly, as in the original stsCPT. In addition, ME-CPT is able to capture the previously mentioned current blocking effects, as shown in the following.

Other NEGF/ME hybrid methods exist in the literature [66,98-101]. For instance, in a recent work [102,103] we have proposed a so-called auxiliary master equation approach (AMEA), whereby a Lindblad equation is introduced which models the leads by a small number of bath sites plus Markovian environments. The AMEA is suited to address steady-state properties of single-impurity problems as encountered in the framework of nonequilibrium dynamical mean field theory $[71,86,102,104-106]$. In contrast, the ME-CPT presented in this work is more appropriate to treat nonlocal self-energy effects which cannot be captured by single-site DMFT.

This paper is organized as follows: After defining the model Hamiltonian in Sec. II, the ME-CPT is introduced in detail in Sec. III. We present results obtained with the improved method for two experimentally realizable devices: (i) in Sec. IV A, an electron-electron interaction based quantum diode, and (ii) and in Sec. IV B, a triple quantum dot ring junction which both feature negative differential conductance (NDC).

For ring systems, extensive ME results and an explanation of the NDC in terms of quantum interference mediated blocking are available in Refs. [44,45].

\section{MODEL}

We consider a model of spin- $\frac{1}{2}$ fermions, having in mind the electronic degrees of freedom of a contacted nanostructure, heterostructure, or a molecular junction. The Hamiltonian consists of three parts:

$$
\hat{\mathcal{H}}=\hat{\mathcal{H}}^{S}+\hat{\mathcal{H}}^{E}+\hat{\mathcal{H}}^{S E} .
$$

(i) The "system" $\hat{\mathcal{H}}^{S}$ represents the interacting central region, i.e., the nanodevice or molecule consisting of single-particle as well as interaction many-body terms. It is described by electronic annihilation/creation operators $f_{i \sigma} / f_{i \sigma}^{\dagger}$ at site $i=$ $\left[1, \ldots, N_{S}\right]$ where $N_{S}$ is typically small and spin $\sigma=\{\uparrow, \downarrow\}$ [107]. We will specify the particular form of $\hat{\mathcal{H}}^{S}$ in the respective results section. (ii) The "environment" Hamiltonian $\hat{\mathcal{H}}^{E}$ describes the two noninteracting electronic leads

$$
\hat{\mathcal{H}}^{E}=\sum_{\lambda=1}^{2} \sum_{k \sigma} \epsilon_{\lambda k \sigma} c_{\lambda k \sigma}^{\dagger} c_{\lambda k \sigma},
$$

where $c_{\lambda k \sigma} / c_{\lambda k \sigma}^{\dagger}$ denote the fermion operators of the infinite size lead $\lambda$ with energies $\epsilon_{\lambda k \sigma}$ and electronic density of states (DOS) $\rho_{\lambda \sigma}(\omega)=\frac{1}{N_{\lambda}} \sum_{k} \delta\left(\omega-\epsilon_{\lambda k \sigma}\right)$ where $N_{\lambda} \rightarrow \infty$ are the number of levels in the leads. The disconnected leads are held at constant temperatures $T_{\lambda}$ and chemical potentials $\mu_{\lambda}$ so that the particles obey the Fermi-Dirac distribution $p_{\lambda}^{\mathrm{FD}}\left(\omega, T_{\lambda}, \mu_{\lambda}\right)$ $[107,108]$. (iii) Finally, the system and the environment are coupled by the single-particle hopping

$$
\hat{\mathcal{H}}^{S E}=\sum_{\lambda=1}^{2} \sum_{i k \sigma}\left(t_{\lambda i k \sigma}^{\prime} f_{i \sigma}^{\dagger} c_{\lambda k \sigma}\right)+\text { H.c. }
$$

\section{MASTER EQUATION BASED CLUSTER PERTURBATION THEORY}

Our goal is to obtain the steady-state transport characteristics of the Hamiltonian $\hat{\mathcal{H}}$ [Eq. (1a)] in a nonequilibrium situation induced by environment parameters such as a bias voltage $V_{B}$ or temperature gradient $\Delta T$. The important step consists in evaluating the steady-state single-particle Green's function in Keldysh space $\widetilde{G}$ in the well-established KeldyshSchwinger nonequilibrium Green's function formalism [109-111]. In general, $\hat{\mathcal{H}}$ is both interacting and of infinite spatial extent. Therefore, explicit evaluation of $\widetilde{G}$ is prohibitive in all but the most simple cases which motivates the introduction of approximate schemes.

One such scheme is CPT [88,89], in which one performs an expansion in a "small" single-particle perturbation, for example, the system-environment coupling $\hat{\mathcal{H}}^{S E}$ of Eq. (1c). The unperturbed Hamiltonian $\hat{\mathcal{H}}^{S}+\hat{\mathcal{H}}^{E}$ can be solved exactly. While in the noninteracting case CPT becomes exact, results obtained in the presence of interaction are approximate and depend on the reference state for the unperturbed system. A common practice within stsCPT [91,93-97] is to use a pure state given by the equilibrium ground state $\left|\Psi_{0}\right\rangle_{S}$ of the disconnected interacting system Hamiltonian $\hat{\mathcal{H}}^{S}$. In a nonequilibrium situation, this is still ambiguous, as it depends on an arbitrary choice of the chemical potential $\mu_{S}$ and/or temperature $T_{S}$ for the interacting finite system.

The goal of this work is to provide an unambiguous and conceptually more rigorous criterion for the choice of the reference state for the interacting central region. Ideally, the reference state is selected such that it resembles best the situation of the coupled system, i.e., for the full Hamiltonian (1a) in the steady state. An appropriate choice in equilibrium is to use the grand-canonical density operator [107] $\hat{\rho}_{g c}^{S}$ as reference state. In this case, $T_{S}$ and $\mu_{S}$ are uniquely determined by the equilibrium situation. Equivalently, $\hat{\rho}_{g c}^{S}$ is given by the steady-state solution of a ME in the Born-Markov approximation (see Sec. III B), when coupling the system to one thermal environment. From this viewpoint, a natural extension to the nonequilibrium situation is to make use of a $\mathrm{ME}$ as well in order to obtain a consistent reference state, given then by the steady-state reduced density operator of the system $\hat{\rho}^{S}$. In this work, a second-order Born-Markov ME is employed, which yields the correct zeroth-order reduced density operator $\hat{\rho}^{S}$ (adjusted to $\hat{\mathcal{H}}^{S E}$ ) [112,113]. Subsequently, $\hat{\mathcal{H}}^{S E}$ is included within the CPT approximation $[88,89]$ in order to obtain improved results for the Green's function and in turn for the transport observables.

In summary, the ME-CPT method consists of the following three main steps, analogous to a standard CPT treatment:

(1) Decompose the whole system into a small interacting central region (system) and noninteracting leads of infinite size (environment) [see $\hat{\mathcal{H}}^{S}$ and $\hat{\mathcal{H}}^{E}$ in Eq. (1a)].

(2) The step introduced in this work is to solve a ME for the system in order to obtain the reduced density operator 
$\hat{\rho}^{S}$, which serves as a reference state to calculate the cluster (retarded) Green's function [114]

$$
g_{i j \sigma}^{R}(\tau)=-i \theta(\tau) \operatorname{tr}\left\{\hat{\rho}^{S}\left[f_{i \sigma}(\tau), f_{j \sigma}^{\dagger}\right]_{+}\right\} .
$$

(3) Reintroduce the system-environment coupling $\hat{\mathcal{H}}^{S E}$ perturbatively [see Sec. III A and Eq. (4)] to determine the Green's function of the coupled system.

\section{A. Steady-state cluster perturbation theory}

Here, we briefly recall the main, well-established CPT concepts and equations, as this is the starting point for the formalism presented in this work. For an in-depth discussion of CPT [115] and its nonequilibrium extension we refer the reader to the literature $[90,92,94,96]$.

The central element of stsCPT is the steady-state singleparticle Green's function in Keldysh space [116]

$$
\widetilde{G}=\left(\begin{array}{cc}
G^{R} & G^{K} \\
0 & G^{A}
\end{array}\right),
$$

where $R$ denotes the retarded, $A$ the advanced, and $K$ the Keldysh component. In the present formalism, $G^{R / A / K}$ become matrices in the space of cluster sites and depend on one energy variable $\omega$ since time translational invariance applies in the steady state.

As explained above, in order to compute $\widetilde{G}(\omega)$ within stsCPT one partitions $\hat{\mathcal{H}}$ [Eq. (1a) in real space] into individually exactly solvable parts, in this case, the system $\hat{\mathcal{H}}^{S}$ and the environment $\hat{\mathcal{H}}^{E}$, which leaves the coupling Hamiltonian $\mathcal{H}^{S E}$ as a perturbation. The single-particle Green's function of the disconnected Hamiltonian is denoted by $\widetilde{g}(\omega)$, which obviously does not mix the disconnected regions. For the noninteracting environment, the respective block entries of $\tilde{g}(\omega)$ are available analytically $[93,117]$. For the interacting part, the respective entries of $\tilde{g}(\omega)$ are calculated via the Lehmann representation with respect to the reference state. This can be computed, e.g., based on the band Lanczos method [118-120].

The full steady-state Green's function in the CPT approximation is found by reintroducing the intercluster coupling perturbatively

$$
\widetilde{G}(\omega)^{-1}=\widetilde{g}(\omega)^{-1}-\tilde{M} ; \quad M^{R}=M^{A}=M, M^{K}=0,
$$

where we denote by the matrix $M$ the single-particle Wannier representation of $\hat{\mathcal{H}}^{S E}$. CPT is equivalent to using the self-energy $\widetilde{\Sigma}$ of the disconnected Hamiltonian as an approximation to the full self-energy. Therefore, the quality of the approximation can in principle be systematically improved by adding more and more sites of the leads to the central cluster. However, in doing so the complexity for the exact solution of the central cluster grows exponentially. Independent of the reference state, this scheme becomes exact in the noninteracting limit.

\section{B. Born-Markov equation for the reference state}

In the following, we outline how to obtain the reference state $\hat{\rho}^{S}$ by using a Born-Markov-secular (BMS-ME) or more generally a Born-Markov ME (BM-ME) [74-79]. Although this approach is standard, for completeness we present here the main aspects and notation. We loosely follow the treatment of Refs. [39,77,78].

The real-time $\tau$ evolution of the full many-body density matrix $\hat{\rho}$ is given by the von Neumann equation $\dot{\hat{\rho}}=-i[\hat{\mathcal{H}}, \hat{\rho}]_{-}$ [74]. Typically, the large size of the Hilbert space of $\hat{\mathcal{H}}$ prohibits the full solution in the interacting case. One thus considers the weak coupling limit $\left|\hat{\mathcal{H}}^{S E}\right| \ll\left|\hat{\mathcal{H}}^{E}\right|$ and performs a perturbation theory in terms of $\left|\hat{\mathcal{H}}^{S E}\right|[79,121]$.

In the usual way, one obtains an equation for the reduced many-body density matrix of the system $\hat{\rho}^{S}(\tau)=$ $\operatorname{tr}_{E}\{\hat{\rho}\}$ by working in the interaction picture $\hat{\rho}_{I}(\tau)=$ $e^{+i\left(\hat{\mathcal{H}}^{S}+\hat{\mathcal{H}}^{E}\right) \tau} \hat{\rho}(0) e^{-i\left(\hat{\mathcal{H}}^{S}+\hat{\mathcal{H}}^{E}\right) \tau}$ with respect to the coupling Hamiltonian [Eq. (1c)]. One then performs three standard approximations: (i) Within the Born approximation, valid to lowest order in $\left|\hat{\mathcal{H}}^{S E}\right|$, the density matrix is factorized $\hat{\rho}_{I}(\tau) \approx \hat{\rho}_{I}^{S}(\tau) \otimes \hat{\rho}_{I}^{E}$. Furthermore, the environment $\hat{\rho}_{I}^{E}$ is assumed to be so large that it is not affected by $\left|\hat{\mathcal{H}}^{S E}\right|$ and thus independent of time. (ii) The Markov approximation implies a memoryless environment, that is, the system density matrix varies much slower in time than the decay time of the environment correlation functions $C_{\alpha \beta}(\tau)$. Upon transforming back to the Schrödinger picture, this yields the BM-ME, which is time local, preserves trace and Hermiticity, and depends on constant coefficients. (iii) To obtain an equation of Lindblad form which also preserves positivity, one typically employs the secular approximation, which averages over fast oscillating terms, yielding the BMS-ME [77,122,123],

The system-environment coupling can be quite generally written in the form $\hat{\mathcal{H}}^{S E}=\sum_{\alpha} \hat{S}_{\alpha} \otimes \hat{E}_{\alpha}$, with $\hat{S}_{\alpha}=\hat{S}_{\alpha}^{\dagger}$ and $\hat{E}_{\alpha}=\hat{E}_{\alpha}^{\dagger}$. This Hermitian form is convenient for further treatment.The tensor product form can be achieved even for fermions by a Jordan-Wigner transformation [78] (see Appendix B). For our coupling Hamiltonian [Eq. (1c)] and particle-number-conserving systems, the coupling operators take the form

$$
\begin{array}{ll}
\hat{S}_{1 i \sigma}=\frac{1}{\sqrt{2}}\left(f_{i \sigma}+f_{i \sigma}^{\dagger}\right), & \hat{E}_{1 \lambda i \sigma}=\frac{1}{\sqrt{2}}\left(c_{\lambda i \sigma}+c_{\lambda i \sigma}^{\dagger}\right), \\
\hat{S}_{2 i \sigma}=\frac{i}{\sqrt{2}}\left(f_{i \sigma}-f_{i \sigma}^{\dagger}\right), & \hat{E}_{2 \lambda i \sigma}=\frac{i}{\sqrt{2}}\left(c_{\lambda i \sigma}-c_{\lambda i \sigma}^{\dagger}\right) .
\end{array}
$$

In the energy eigenbasis of the system Hamiltonian $\hat{\mathcal{H}}^{S}|a\rangle=\omega_{a}|a\rangle$, the BM-ME in the Schrödinger representation reads as [114]

$$
\begin{aligned}
\dot{\hat{\rho}}^{S}(\tau)= & -i\left[\hat{\mathcal{H}}^{S}+\hat{\mathcal{H}}^{L S}, \hat{\rho}^{S}(\tau)\right]_{-}+\sum_{a b c d} \Xi_{a b, c d} \\
& \times\left(|a\rangle\left\langle b\left|\hat{\rho}^{S}(\tau)\right| d\right\rangle\langle c|-\frac{1}{2}\left[|d\rangle\langle c|| a\rangle\langle b|, \hat{\rho}^{S}(\tau)\right]_{+}\right),
\end{aligned}
$$

with

$$
\Xi_{a b, c d}=\sum_{\alpha \beta} \xi_{\alpha \beta}\left(\omega_{b a}, \omega_{d c}\right)\left\langle a\left|\hat{S}_{\beta}\right| b\right\rangle\left\langle c\left|\hat{S}_{\alpha}\right| d\right\rangle^{*},
$$

where $\omega_{b a}=\omega_{b}-\omega_{a}$. The Lamb-shift Hamiltonian $\hat{\mathcal{H}}^{\text {LS }}$ and the environment functions $\xi_{\alpha \beta}\left(\omega_{1}, \omega_{2}\right)$ are defined in Appendix A. When employing the secular approximation, the terms in the BMS-ME simplify and in Eq. (7) one can replace 
$\xi_{\alpha \beta}\left(\omega_{b a}, \omega_{d c}\right) \rightarrow \xi_{\alpha \beta}\left(\omega_{b}-\omega_{a}\right) \delta_{\omega_{b}-\omega_{a}, \omega_{d}-\omega_{c}}$. Due to the secular approximation, the BMS-ME can only lead to interference between degenerate states. The more general BM-ME also couples nondegenerate states at the cost of losing the Lindblad structure of the ME (see Sec. IV B and [39]).

\section{Single-particle Green's function}

As discussed above, for ME-CPT, the Green's function $\widetilde{g}(\omega)$ of the isolated system is evaluated from the reference state $\hat{\rho}^{S}$. The retarded component (2) takes the explicit form

$$
\begin{aligned}
g_{i j(\sigma)}^{R}(\omega)= & \sum_{a b c} \rho_{a b}^{S}\left(\frac{\left\langle b\left|f_{i \sigma}\right| c\right\rangle\left\langle c\left|f_{j \sigma}^{\dagger}\right| a\right\rangle}{\omega+i 0^{+}-\left(\omega_{c}-\omega_{b}\right)}\right. \\
& \left.+\frac{\left\langle b\left|f_{j \sigma}^{\dagger}\right| c\right\rangle\left\langle c\left|f_{i \sigma}\right| a\right\rangle}{\omega+i 0^{+}-\left(\omega_{a}-\omega_{c}\right)}\right),
\end{aligned}
$$

where $i, j$ denote indices of system sites. The advanced component follows from $g^{A}=\left(g^{R}\right)^{\dagger}$ and the Keldysh component $g^{K}$ of the finite, unperturbed system is not relevant for the CPT equation (4). Once $\widetilde{g}$ is obtained, the full Green's function is again approximately obtained within CPT by Eq. (4). Notice that for $U=0, \widetilde{G}$ is independent of the reference state, which is why stsCPT, stsVCA, as well as ME-CPT coincide (and become exact) in the noninteracting case.

\section{Numerical implementation}

From a numerical point of view, the two main steps are to first obtain the reference state $\hat{\rho}^{S}$ by solving the ME and then to evaluate the Green's functions using Eqs. (8) and (4). For the solution of the BM-ME [Eq. (6)] one needs to carry out the following: (i) Full diagonalization of the interacting system Hamiltonian which is done in LAPACK, making use of the block structure in $\hat{N}$ and $\hat{S}^{z}$. (ii) Evaluation of the coefficients of the BM-ME in Eq. (6), which involves coupling matrix elements $\left\langle a\left|\hat{S}_{\alpha}\right| b\right\rangle$ and numerical integration of the bath correlations functions (see Appendixes $\mathrm{A}$ and $\mathrm{C}$ ), for which an adaptive Gauss-Kronrod scheme is employed. (iii) The steady state $\hat{\rho}^{S}$ is finally obtained from the unique eigenvector with eigenvalue zero of Eq. (6), which we determine by a sparse Arnoldi diagonalization. Again, a block structure is related to $\hat{N}$ and $\hat{S}^{z}$. The numerical effort for the exact diagonalization scales with the size of the Hilbert space, and therefore exponentially with the system size $N_{S}$. In the second major step, the Green's function of the disconnected system is calculated by Eq. (8). Finally, the ME-CPT Green's function $\widetilde{G}(\omega)$ is found using Eq. (4). We outline how to evaluate observables within ME-CPT and the ME in Appendix D.

\section{RESULTS}

In this section, we present results obtained from the MECPT approach. In all calculations, except those in Sec. IV B, the secular approximation is applied for the reference state $\hat{\rho}^{S}$. The main improvements of ME-CPT with respect to bare BMS-ME are (i) the inclusion of lead-induced broadening effects, (ii) the correct $U=0$ limit, and (iii) a correction for effects missed by an improper treatment of quasidegenerate states in the BMS-ME (see following). In comparison to the

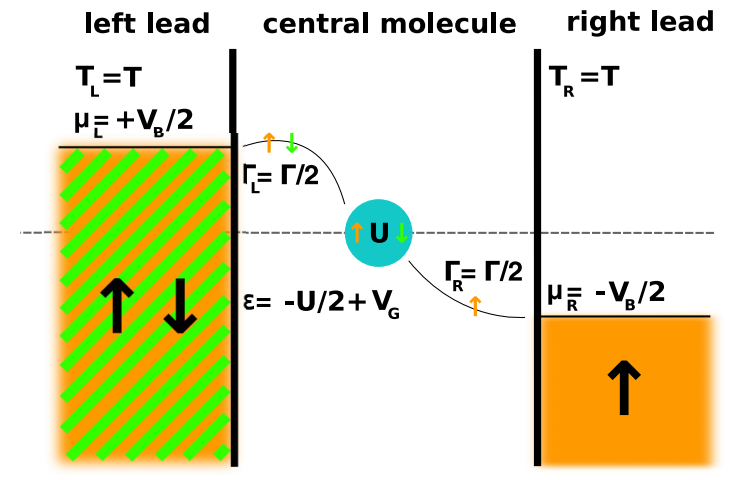

FIG. 1. (Color online) Quantum dot diode: Schematic representation (see Sec. IV A). Single quantum dot with Hubbard interaction $U$ and gate voltage $V_{G}$ (particle-hole symmetric at $V_{G}=0$ ), coupled via $\Gamma_{\mathrm{L} / \mathrm{R}}=\frac{\Gamma}{2}$ to a left and right lead. The right lead is fully polarized, i.e., only spin- $\uparrow$ DOS is present. An external bias voltage $V_{B}$ shifts the chemical potentials by $\mu_{\mathrm{L} / \mathrm{R}}= \pm \frac{V_{B}}{2}$. The leads are in the wide band limit and at the same temperature $T$.

previous "standard" stsCPT, ME-CPT also captures current blocking effects, which are discussed in detail in Refs. [38,39] within a ME treatment.

\section{A. Quantum dot diode}

We first discuss a quite simple model system: a quantum diode based on electron-electron interaction effects. Figure 1 depicts this junction consisting of a single interacting orbital described by a Hubbard interaction and an onsite term to allow for a gate voltage $V_{G}$ [124]:

$$
\hat{\mathcal{H}}^{S}=U\left(\hat{n}_{\uparrow}^{f}-\frac{1}{2}\right)\left(\hat{n}_{\downarrow}^{f}-\frac{1}{2}\right)+V_{G} \sum_{\sigma} \hat{n}_{\sigma}^{f},
$$

where $\hat{n}_{\sigma}^{f}=f_{\sigma}^{\dagger} f_{\sigma}$. The environment Eq. (1b) consists of two spin-dependent, conducting leads. We model both the left (L) and the right $(\mathrm{R})$ leads by a flat DOS with local retarded singleparticle Green's function [117] $g_{\mathrm{L} / \mathrm{R}}^{R}(\omega)=-\frac{1}{2 D} \ln \left(\frac{\omega+i 0^{+}-D}{\omega+i 0^{+}+D}\right)$, with a half-bandwidth $D$ much larger than all other energy scales in the model, mimicking a wide band limit. We keep both leads at the same temperature $T_{\mathrm{L}}=T_{\mathrm{R}}=T$ and at chemical potentials $\mu_{\mathrm{L}}=-\mu_{\mathrm{R}}=\frac{V_{B}}{2}$ corresponding to a symmetrically applied bias voltage $V_{B}$. The right lead is fully spin polarized, i.e., tunneling of one spin species $(\downarrow)$ into the right lead is prohibited while both spin species can tunnel to the left lead. The system is coupled to the two leads via a single-particle hopping amplitude $t^{\prime}$ in $\hat{\mathcal{H}}^{S E}$ [Eq. (1c)] which results in a lead broadening parameter of $\Gamma_{\mathrm{L}}^{\uparrow}=\Gamma_{\mathrm{L}}^{\downarrow}=\Gamma_{\mathrm{R}}^{\uparrow}=\frac{\Gamma}{2}=\pi\left|t^{\prime}\right|^{2} \frac{1}{2 D}$ [Eq. (C1)] and $\Gamma_{R}^{\downarrow} \equiv 0$. We use $\Gamma$ without an argument for $\Gamma(\omega=0)$ as defined in Eq. (C1). For ME-CPT we use $\mathcal{H}^{S E}$ [see Eq. (1c)] as perturbation.

Such a system could be realized in (i) a "metal-artificial atom-half-metallic ferromagnet" [125] nanostructure where spin- $\uparrow$ DOS is present at the Fermi energy while the respective spin- $\downarrow$ DOS is zero; (ii) a graphene nanostructure [30,31] with ferromagnetic cobalt electrodes [32]; (iii) a one-dimensional optical lattice of ultracold fermions in a quantum simulator [126] where the hopping of spin- $\downarrow$ particles into the right 

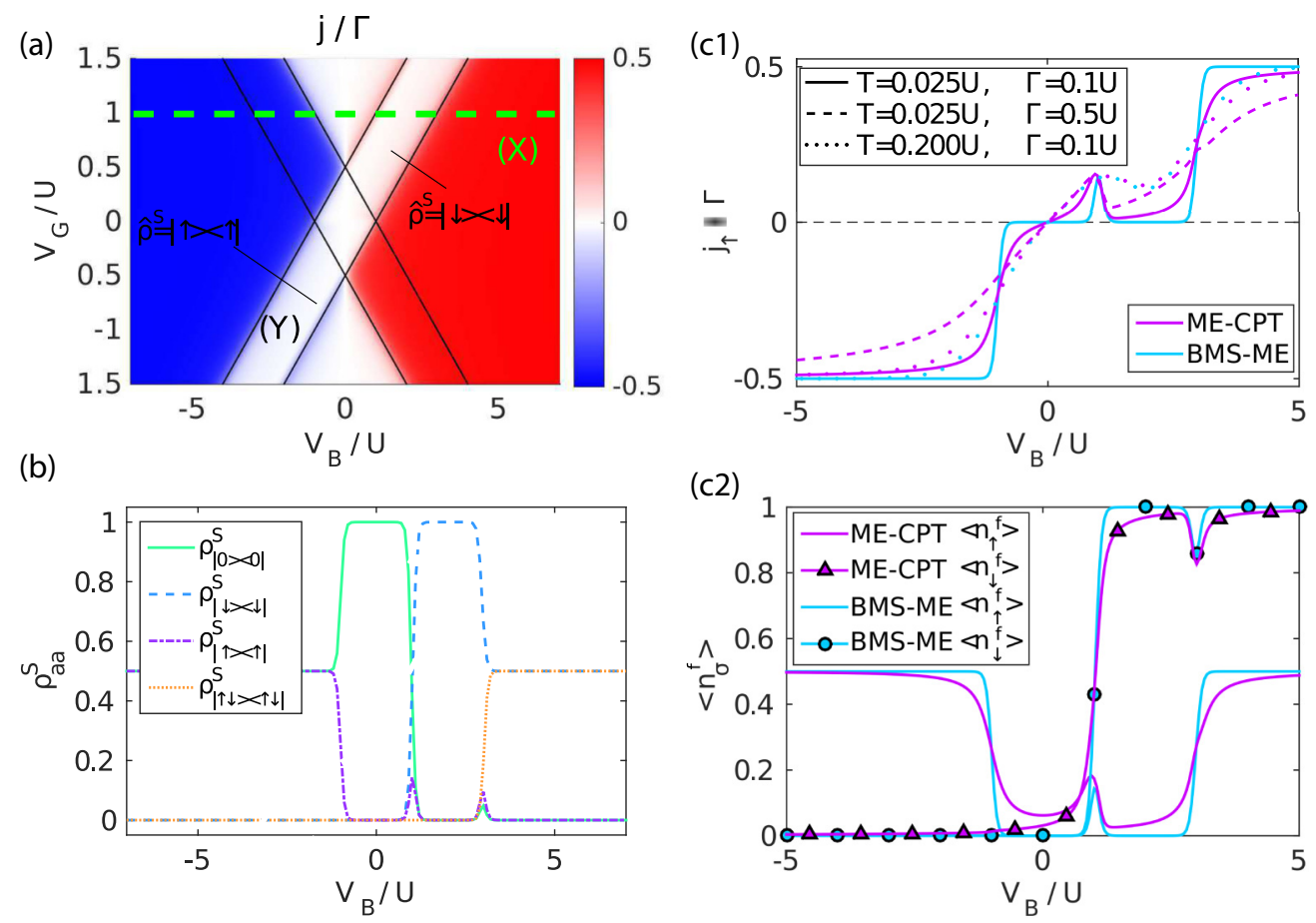

FIG. 2. (Color online) Quantum dot diode: (a) Stability diagram, based on the total current $j=\left\langle j_{\uparrow}\right\rangle+\left\langle j_{\downarrow}\right\rangle$ as a function of bias voltage $V_{B}$ and gate voltage $V_{G}$, obtained within ME-CPT. Note that $\left\langle j_{\downarrow}\right\rangle \equiv 0$. Results are depicted for $T=0.025 U$ and $\Gamma=0.1 U$. (Y) marks the current blocking region. The green dashed line $(\mathrm{X})$ at $V_{G}=U$ indicates the parameter regime for the panels (b) and (c). (b) Diagonal part of the reduced density matrix $\rho_{a a}^{S}$ obtained by BMS-ME. (c1) Spin- $\uparrow$ current $j_{\uparrow}$ within ME-CPT compared to BMS-ME. Solid lines are for the same parameters as line (X) in panel (a). Blue dashed and solid lines for BMS-ME are indistinguishable. (c2) Spin-resolved densities $\left\langle n_{\uparrow}\right\rangle$ and $\left\langle n_{\downarrow}\right\rangle$ for the same parameters as in panel (c1) (see solid lines in the legend).

reservoir is suppressed. For all three systems, spin- $\downarrow$ particles cannot reach the right lead, in the first two due to a vanishing DOS, in the third one due to a vanishing tunneling amplitude.

We consider parameters such that the junction is operated in a single-electron transistor (SET) regime [36], i.e., temperatures above the Kondo temperature [48]. In this regime, we expect an interaction-induced-magnetization-mediated blocking due to the fact that the system fills up with spin- $\downarrow$ particles. On the one hand, they cannot escape, yielding a vanishing spin- $\downarrow$ current, and on the other hand, they suppress the spin- $\uparrow$ occupation, at finite repulsive interaction $U$, resulting also in a vanishing spin- $\uparrow$ current [45].

Figure 2(a) shows the ME-CPT stability diagram of the interacting system in the $V_{B}-V_{G}$ plane. When applying a particle-hole transformation for all particles, leads, and system, along with $t^{\prime} \rightarrow-t^{\prime}$ we easily find the symmetry properties

$$
\begin{gathered}
j\left(-V_{B},-V_{G}\right)=-j\left(V_{B}, V_{G}\right), \\
\left\langle n_{\sigma}^{f}\right\rangle\left(-V_{B},-V_{G}\right)=1-\left\langle n_{\sigma}^{f}\right\rangle\left(V_{B}, V_{G}\right) .
\end{gathered}
$$

From the continuity equation it is clear that only spin- $\uparrow$ steady-state current can flow which limits the maximum current to $\frac{\Gamma}{2}$. The energies $\omega_{N}$ of the isolated quantum dot can be labeled by the total particle number $N$ and are for $V_{G}=U$ given by $\omega_{0}=0, \omega_{1}=\frac{1}{2} U$, and $\omega_{2}=2 U$. This gate voltage corresponds to the dashed line, marked by (X) in Fig. 2(a). The corresponding energy differences $\Delta_{01}=0.5 \mathrm{U}$ between the single-occupied and the empty dot and $\Delta_{12}=1.5 \mathrm{U}$ between double-occupied and single-occupied dot are associated with a further transport channel opening as soon as the bias $V_{B}$ reaches twice their values. The ME-CPT result for the current exhibits the well-known Coulomb diamond [36] close to $V_{B}=0$ and $V_{G}=0$, where current is hindered because all system energies are far outside the transport window $\pm \frac{V_{B}}{2}$ [see Eq. (D2)]. At $V_{G}=0$ a current sets in at $\frac{V_{B}}{2}= \pm\left|\frac{U}{2}\right|$, i.e., when transport across the system's single-particle level becomes allowed. The point, at which the current sets in, shifts with $V_{G}$ linearly to higher-bias voltages. This transition is broadened $\propto \max (\Gamma=0.1 U, T=0.025 U)$. However, not only the transport window and possible excitations in the system energies determine the current-voltage characteristics. The particular occupation of the system states may lead to more complicated effects, such as current blocking.

Our first main result is that in contrast to stsCPT the blocking is correctly reproduced in ME-CPT. The current blocking is visible in Fig. 2(a) in region (Y) [see also the detailed data in subplot (c1)]. It is asymmetric in $V_{B}$ and therefore responsible for the rectifying behavior for $\left|V_{G}\right|>$ $\left|\frac{U}{2}\right|$. This feature is easily understood from the plots of the spin-resolved densities in Fig. 2(c2). In the region of interest, for positive $V_{B},\left\langle n_{\downarrow}\right\rangle=1$ which hinders spin- $\uparrow$ particles from the left lead to enter the system, due to the repulsive interaction $U$ and suppresses the current. For negative $V_{B}$, the situation is reversed. A direct computation of the current in the framework of BMS-ME (see Appendix D 2) also predicts the blocking, which is however not the case if we use stsCPT based on the zero-temperature ground state $\left|\Psi_{0}\right\rangle_{S}$. The blocking is evident in Fig. 2(b), where we observe that in the blocking regime, 
TABLE I. Comparison of steady-state cluster perturbation theory (stsCPT), the Born-Markov-secular master equation (BMS-ME), and the quantum master equation based stsCPT (ME-CPT) with respect to their ability to capture temperature $(T)$ or lead $(\Gamma)$ induced level broadening, current blocking, and whether the noninteracting limit is fulfilled.

\begin{tabular}{lcccc}
\hline \hline Method & \multirow{2}{*}{ broadening } & $\Gamma$ broadening & Blocking & $U=0$ \\
\hline stsCPT & Yes & Yes & No & Exact \\
BMS-ME & Yes & No & Yes & Approx. \\
ME-CPT & Yes & Yes & Yes & Exact \\
\hline \hline
\end{tabular}

the reduced density is $\rho_{S}=|\downarrow\rangle\langle\downarrow|$. Independent of the value of $U>0$, the blocking sets in at the same values of $V_{B}$ in ME-CPT and BMS-ME. Figure 2(c1) shows that within BMS-ME this regime is entered after a $U$-independent hump in the current, while within ME-CPT the hump is broader and weakly $U$ dependent. The current blocking disappears at a bias voltage $V_{B} \propto U$ in both methods. Immediately apparent are the much broader features in ME-CPT, which leads to a less pronounced effect in contrast to the total blocking predicted by BMS-ME. In BMS-ME, the broadening parameter $\Gamma$ enters merely as prefactor of the current, and broadening is solely induced by the temperature. This temperature-induced broadening is correctly taken into account in both methods. For $T>\Gamma$, the latter dominates and the ME-CPT results are similar to the plain BMS-ME solution. A comparison of the three methods is given in Table I. In this simple model, the blocking can be captured even by a straightforward steadystate mean-field theory in the Keldysh Green's function with self-consistently determined spin densities or in stsVCA. This is not the case for the more elaborate system studied in the next section.

\section{B. Triple quantum dot}

In this section, we discuss a more elaborate model system: a triple quantum dot ring junction which features negative differential conductance (NDC) based on electron-electron interaction effects mediated by quantum interference due to degenerate states as outlined in detail in Refs. [44,45].
Figure 3(a) depicts the triple quantum dot ring junction, described by the following Hubbard Hamiltonian [127]:

$$
\begin{aligned}
\hat{\mathcal{H}}^{S}= & \sum_{i=1}^{3} U\left(\hat{n}_{i \uparrow}^{f}-\frac{1}{2}\right)\left(\hat{n}_{i \downarrow}^{f}-\frac{1}{2}\right)+V_{G} \sum_{i=1}^{3} \sum_{\sigma} \hat{n}_{i \sigma}^{f} \\
& +t \sum_{\langle i j\rangle} \sum_{\sigma} f_{i \sigma}^{\dagger} f_{j \sigma} .
\end{aligned}
$$

In addition to the model parameters described in Sec. IV A, a nearest-neighbor $\langle i j\rangle$ hopping $t$ is present. The environment [Eq. (1b)] and coupling [Eq. (1c)] are now both symmetric in spin. Moreover, we use $\mu_{\mathrm{L}}=-\mu_{\mathrm{R}}=\frac{V_{B}}{2}, T=T_{\mathrm{L}}=T_{\mathrm{R}}$, and $\Gamma_{\mathrm{L}}=\Gamma_{\mathrm{R}}=\frac{\Gamma}{2}=\pi\left|t^{\prime}\right|^{2} \frac{1}{2 D}$.

Such a junction can be experimentally realized: (i) via local anodic oxidation (LAO) on a GaAs/AlGaAs heterostructure [28] which enables tunable few electron control [29]; (ii) in a graphene nanostructure [30,31]. Experimentally, the stability diagram has been explored [33] alongside characterization and transport measurements [28,34,35]. The negative differential conductance has been observed in a device aimed as a quantum rectifier [128]. Theoretically, the study of the nonequilibrium behavior of such a device has become an active field recently [44,45,129-135].

We investigate transport properties for values of the parameters such that the junction is in a single-electron transistor (SET) regime [36], i.e., temperatures above the Kondo temperature [48]. In this regime, we expect an interaction-induced-quantum-interference-mediated blocking as discussed in Refs. [44,45]. The rotational symmetry ensures degenerate eigenstates labeled by a quantum number of angular momentum. In situations where these degenerate states participate in the transport they provide two equivalent pathways through the system and lead to quantum interference [44]. The blocking sets in at a bias voltage, where the degenerate states start to participate in the transport. It then becomes possible that a superposition is selected which forms one state with a node at the right lead. In the long-time limit, this state will be fully occupied while the other one will be empty due to Coulomb repulsion, for reasons very similar to those discussed in the previous section $[38,39]$. (a)

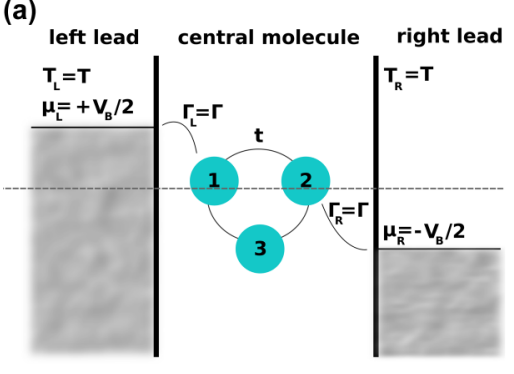

(b1)

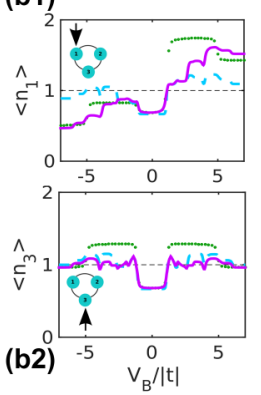

(c)

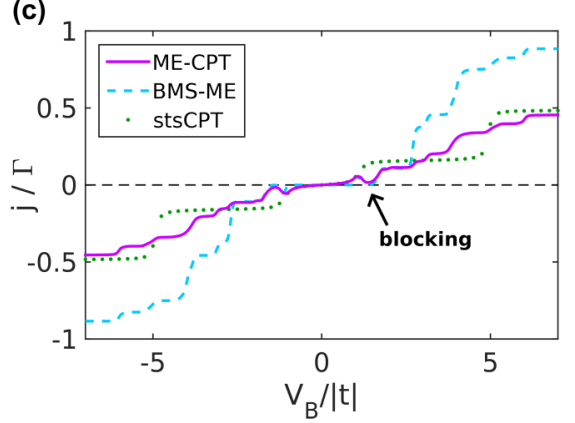

FIG. 3. (Color online) Triple quantum dot: (a) Schematic representation, see Sec. IV B. System Hamiltonian as defined in Eq. (9). Site 1 couples to the left lead and site 2 to the right one, both with $\Gamma_{\mathrm{L} / \mathrm{R}}=\frac{\Gamma}{2}$. The leads are held at the same temperature $T_{\mathrm{L} / \mathrm{R}}=T$ and the chemical potentials $\mu_{\mathrm{L} / \mathrm{R}}= \pm \frac{V_{B}}{2}$ are shifted by the bias voltage. (b) Local charge density $\left\langle n_{i}\right\rangle$ as a function of bias voltage $V_{B}$. The results are obtained by ME-CPT, BMS-ME, and stsCPT [see color code of panel (c)]. (c) Total current $j=\sum_{\sigma}\left\langle j_{L 1 \sigma}\right\rangle$ into the system at site 1 as a function of bias voltage $V_{B}$. Results, shown in panels (b) and (c), are for $U=2|t|, T \approx 0.02|t|, \Gamma=0.1|t|$, and $V_{G}=0$, corresponding to line (X) in Fig. 4 . 


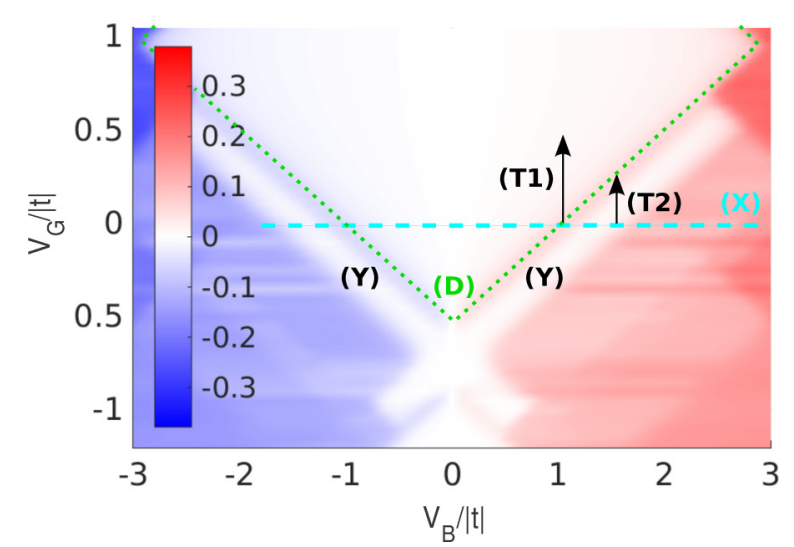

FIG. 4. (Color online) Triple quantum dot: Stability diagram. Total current entering the system as a function of bias voltage $V_{B}$ and gate voltage $V_{G}$, obtained within ME-CPT. The blocking region is indicated by (Y), the Coulomb diamond by (D). The two arrows (T1) and (T2) mark two device operation modes as discussed in the text. All results are for $U=2|t|, T=0.02|t|$, and $\Gamma=0.1|t|$. Dashed line $(\mathrm{X})$ for $V_{G}=0$ marks the parameter region depicted in Fig. 3(c).

The steady-state charge distribution and current-voltage characteristics of the interacting triple quantum dot are presented in Figs. 3(b) and 3(c) in a wide bias voltage window. The current, depicted in Fig. 3(c), in general increases in a stepwise manner and is fully antisymmetric with respect to the bias voltage direction. A blocking effect occurs at $V_{B} \approx 1.5|t|$ as can be observed in the BMS-ME and ME-CPT data. The previous version of stsCPT based on the pure zerotemperature ground state $\left|\Psi_{0}\right\rangle_{S}$ misses this region of NDC. In contrast to the simpler model presented in the previous section, a self-consistent mean-field solution does not capture the blocking effects correctly in this more elaborate system. The BMS-ME solution shows many more steps in the current than the stsCPT one, which is due to transitions in the reference state $\hat{\rho}^{S}$ of the central region. The ME-CPT results in general follow these finer steps, correcting their width to incorporate also lead-induced broadening effects in addition to the pure temperature broadening. As can be seen in Fig. 3(b1), ME-CPT predicts a large charge increase at the site connected to the high-bias lead. Note that the charge density at site 2 , which is connected to the right lead, is simply $\left\langle n_{2}\right\rangle\left(V_{B}\right)=\left\langle n_{1}\right\rangle\left(-V_{B}\right)$. The charge density at site 3 is symmetric with respect to the bias voltage origin.

Next, we study the impact of a gate voltage on the blocking. Results obtained by ME-CPT are depicted as stability diagram in Fig. 4. Upon increasing $\left|V_{G}\right|$, the onset of the blocking shifts linearly to higher $V_{B}(\mathrm{Y})$. We find a Coulomb diamond for $2 V_{G} \gtrsim V_{B}-|t|$ (D). Upon increasing the bias voltage out of the Coulomb diamond [see, e.g., line (X)], a current sets in but is promptly hindered by the blocking so that the current diminishes after a hump of width $\propto \max (T, \Gamma)$. Interestingly, this device could be operated as a transistor in two fundamentally different modes. In mode (T1), at a source-drain voltage of $\approx|t|$ the current is on for a gate voltage of $V_{G}=0$ and off for $V_{G} \approx 0.5|t|$ due to the Coulomb blockade. In mode (T2), at a source-drain voltage of $\approx 1.5|t|$ the current is off for a gate voltage of $V_{G}=0$ due to quantum interference-mediated blocking and on for $V_{G}=0.25|t|$.

Next, we discuss the current characteristics in the vicinity of the blocking in more detail, as well as the impact of the interaction strength $U$. The first row of Fig. 5 shows the total current through the device for different values of $U$. The blocking region shifts to lower bias voltages with increasing $U$. As discussed earlier, structures in the BMS-ME results are only broadened by temperature effects in the steady-state density (compare, e.g., the width of the structures in the local density in the second row of Fig. 5), while ME-CPT additionally takes into account the finite lifetime of the quasiparticles due to the coupling to the leads, given by $1 / \Gamma$. This can be seen by solving Eq. (4) for the local Green's function at device sites. Especially for higher lead broadening $\Gamma$ this gives rise to significant differences in the ME-CPT results compared to the BMS-ME data. From the bottom row of Fig. 5 we see that, before the blocking regime is entered, the steady state changes from a pure $N=2$ state to a mixed $N=2 / N=3$ state at the hump in the current. Obviously, blocking arises because the system reaches a pure $N=3$ state for $U=2|t|$ and $U=3|t|$ at $V_{B} \approx 1.4|t|$. For $U=|t|$ the current is only partially blocked because the contribution of the $N=2$ state is not fully suppressed. For all $U$ values, however, we find NDC. As far as the ME-CPT current is concerned, the complete blocking at higher interaction strengths, predicted by BMS-ME, is reduced to a partial blocking due to the lead-induced broadening effects in ME-CPT. Although $\rho_{a b}^{S}$ changes significantly twice in the blocking region (for $U=2$ and 3 ), the charge density $\left\langle n_{i}\right\rangle$ just increases once from $\left\langle n_{1}\right\rangle \approx 0.75$ to $\left\langle n_{1}\right\rangle \approx 1$.

Details of the steady-state dynamics are provided in Fig. 6 . Before the blocking region is entered $\left(V_{B}=0.4|t|\right)$ the system is in a pure state with $N=2$, which corresponds to the zero-temperature ground state $\left|\Psi_{0}\right\rangle_{S}$ in the $N=2$ sector. Here, the transmission function $\mathcal{T}(\omega)$ [Eq. (D3)] of ME-CPT agrees with the one of stsCPT. A small current is obtained due to the $N=2 \rightarrow 3$ excitation at $\omega \approx 0.55|t|$. Increasing the bias voltage has no influence on the reference state in stsCPT, which therefore remains in the $N=2$ particle sector. Consequently, the transmission function in stsCPT does not change. Only the transport window increases linearly with increasing $V_{B}$. For $V_{B}=1.4|t|$, it includes the peak at $\approx 0.7|t|$ and results in a significant increase in the current obtained in stsCPT (see stsCPT result in Fig. 3). This is in stark contrast to the BMS-ME current, depicted in Fig. 5, which exhibits perfect blocking for $V_{B}=1.4|t|$. The reason for the current blocking is that only two states, both in the $N=3$ sector and doubly degenerate, have significant weight in $\rho_{a b}^{S}$. The ME-CPT solution is based on the modified density matrix and therefore the current is diminished since the next possible excitation is at $\omega \approx 0.9|t|(N=2 \rightarrow 3)$, which is outside the transport window $W(\omega) \approx(-0.7|t|, 0.7|t|)$ [Eq. (D2)]. Due to the leadinduced broadening of $\mathcal{T}(\omega)$ and the temperature-induced broadening of the transport window, the current is however only partially blocked. For $V_{B}=2.4|t|$, this excitation falls into the transport window and the current is no longer blocked. In this case, the state $\rho_{a b}^{S}$ is a mixture of $N=2,3,4$. The dominant excitation responsible for this current is again the ground-state excitation at $\omega \approx 0.55|t|$ from $N=2 \rightarrow 3$. This 

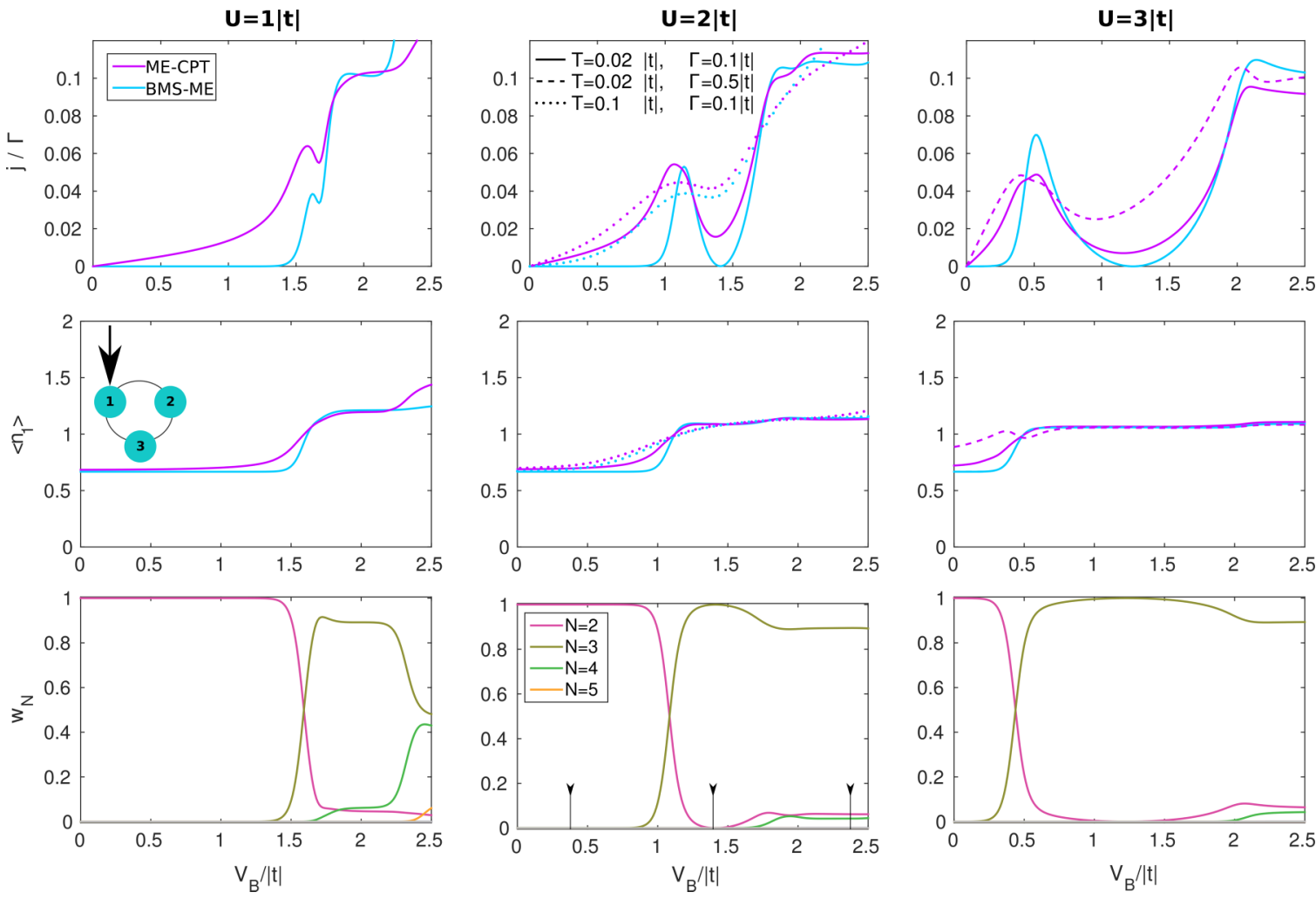

FIG. 5. (Color online) Triple quantum dot: Dependence of the current blocking on the interaction strength $U$. (Top row) Total current $j$ as a function of bias voltage $V_{B}$. (Middle row) Charge density $\left\langle n_{1}\right\rangle$ at site 1 . The color code of the top row is valid. (Bottom row) Summed diagonal elements of the density matrix $w_{N}=\sum_{a \in N} \rho_{a a}^{S}$ per particle number $N$. The black markers in the mid panel $(U=2|t|)$ indicate for which $V_{B}$ detailed results are given in Fig. 6. Solid lines in all panels are for $T=0.02|t|, \Gamma=0.1|t|$, and $V_{G}=0$. Results for $T=0.1|t|$ are depicted in the central panels by dotted lines and those for $\Gamma=0.5|t|$ in the right panels by dashed lines.

is why in this regime the stsCPT current, based on the pure two-particle state is again similar to the ME-CPT current.

Our results on the ME level have been checked with those presented by Begemann et al. in Ref. [38] and Darau et al. in Ref. [39] for a six-orbital ring which shows similar blocking effects. Different types of blocking effects in various parameter regimes have been discussed in detail in a ME framework also for the three-orbital ring by Donarini et al. in Refs. [44,45].

\section{Quasidegenerate states}

Next, we study the reliability of the secular approximation in the case of quasidegeneracy of the isolated energies of the system and benchmark its applicability to create a reference state for ME-CPT. To this end, we apply a second gate voltage that couples only to the third orbital [see Fig. 3 (left)] and leads to an additional term $V_{G, 3} \hat{n}_{3}^{f}$ in the system Hamiltonian. This lifts the degeneracy of states present at $V_{G, 3}=0$ and therefore requires a treatment within the BM-ME (see [39]).

In the following, we discuss the same parameter regime as above. In Fig. 7, we present results obtained using ME-CPT (solid lines) and ME results (dashed lines) for the BMSME (a) and for the BM-ME (b). The ME-CPT results of each panel are obtained using the respective $\mathrm{ME}$. In the BMS-ME data, a very small $\left|V_{G, 3}\right|$ has a drastic effect on the current-voltage characteristics. The blocking present at
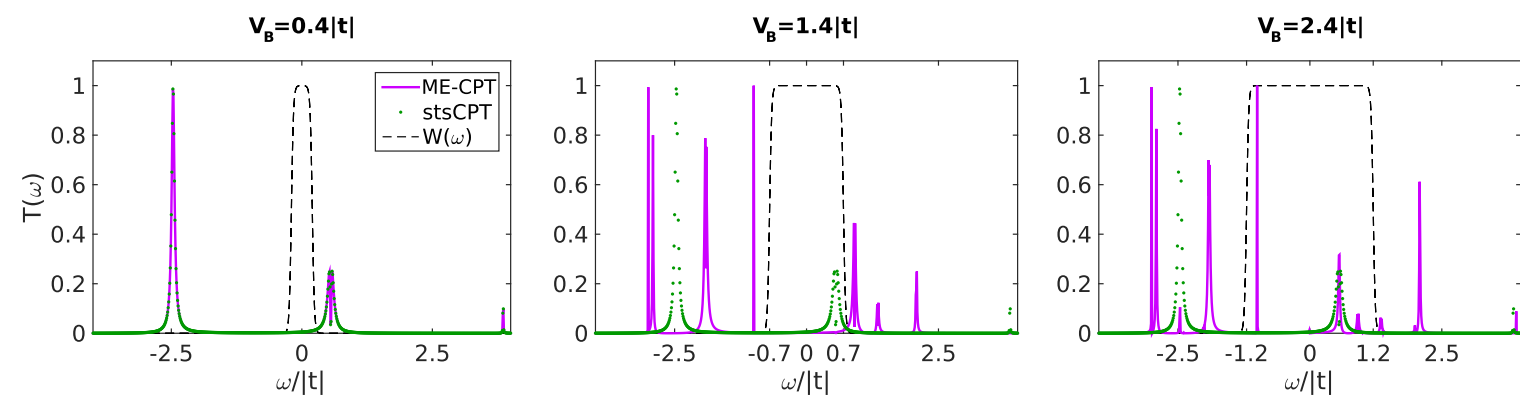

FIG. 6. (Color online) Triple quantum dot: Dynamic transmission function $\mathcal{T}(\omega)$ [Eq. (D3)], as obtained by ME-CPT and stsCPT. Same parameters as in Fig. 5 (bottom mid) at the three indicated bias voltages: $V_{B}=0.4|t|$ (left), $V_{B}=1.4|t|$ (middle), and $V_{B}=2.4|t|$ (right). The temperature-broadened transport window $W(\omega)$ [Eq. (D2)] is depicted as a dashed black line. 
(a) Born-Markov-secular

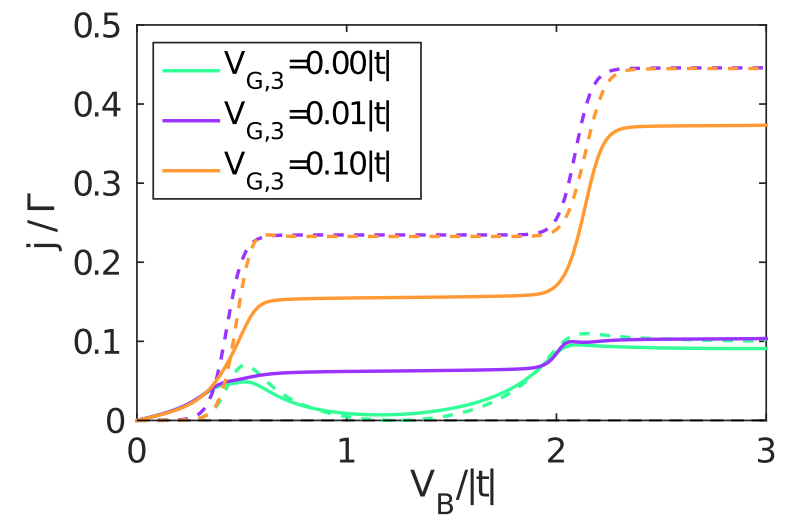

(b) Born-Markov

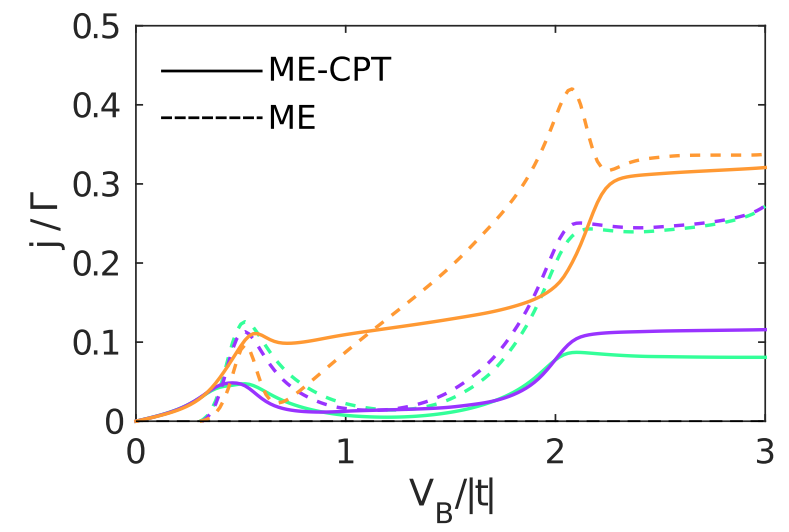

FIG. 7. (Color online) Triple quantum dot: Effects of lifting degeneracies in the system energies by a third gate voltage. Total current $j$ as a function of bias voltage $V_{B}$, for three different gate voltages $V_{G, 3}$ applied to site 3 . Results based on the Born-Markovsecular approximation are compared with those of the Born-Markov approximation. Solid/dashed lines indicate the ME-CPT/BM(s)me result. All results are for $U=3|t|, T=0.02|t|$, and $\Gamma=0.1|t|$.

$V_{G, 3}=0$ is immediately lifted by very small $\left|V_{G, 3}\right|$ and the current jumps to a plateau. For larger $\left|V_{G, 3}\right|$, the current stays on this plateau until further transport channels open up. This "jump" at small $\left|V_{G, 3}\right|$ arises due to the improper treatment of quasidegeneracies in BMS-ME. MeCPT results based on BMS-ME show a smooth change of the currentvoltage characteristics. BM-ME on the other hand correctly accounts for the coupling of the quasidegenerate states and also exhibits a smooth dependence on $V_{G, 3}$. For ME-CPT based on BM-ME we find qualitative similar results to ME-CPT based on BMS-ME, which emphasizes the robustness of the ME-CPT results in general. From this it is apparent that ME-CPT is capable of repairing the decoupling of quasidegenerate states in the BMS-ME to some degree. However, to study blocking effects at quasidegenerate points, it is of advantage to make use of the BM-ME in ME-CPT.

As discussed in Sec. IV C, the BM-ME is not of Lindblad form and does not necessarily result in a positive-definite reduced many-body density matrix $\rho_{a b}^{S}$ in general. Using a not proper density matrix in Eq. (8) may result in noncausal Green's functions when the steady state $\rho_{a b}^{S}$ is obtained from the BM-ME. This can be avoided by using a modified reference state $\rho_{a b}^{S} \rightarrow \rho_{a b}^{S} \Theta\left(\Delta-\left|\omega_{a}-\omega_{b}\right|\right)$, with $\Theta(x)$ the Heaviside step function and $\Delta$ a small quantity, being e.g. $\approx 10^{-6}$, in Eq. (8), which renders the Green's functions causal. This is somewhat an ad hoc approximation and should be seen simply as a way to explore the effects of continuously breaking degeneracy in the problem.

\section{Current conservation}

Finally, we comment on conservation laws in ME-CPT. Within BMS-ME and BM-ME, the current conservation (continuity equation) is always maximally violated in a sense that the current within the system is zero. This is due to the zeroth order $\hat{\rho}^{S}$ as discussed in Appendix D 2. In BMS-ME, the inflow from the left lead into the system, however, always equals the outflow from the system to the right lead. Without the secular approximation, the quantum master equation (BM-ME) is not of Lindblad form and the final many-body density matrix is not guaranteed to be positive definite $[122,123]$. This in turn can lead to slightly negative currents in regions where they are required to be positive by the direction of the bias voltage [77]. Furthermore, the inflow can be slightly different from the outflow.

In the noninteracting case, ME-CPT fully repairs the violation of the continuity equation present in the reference state. For increasing interaction strength, the violation of the continuity equation typically grows also in ME-CPT. In particular, the overall symmetry of the current stays intact (in our case, inflow equals outflow), while the current on bonds between interacting sites does not exactly match the current between noninteracting sites. This typically small violation of the continuity equation can be attributed to the violation of Ward identities $[136,137]$ in the nonconserving approximation scheme of CPT $[138,139]$.

\section{SUMMARY AND CONCLUSIONS}

We improved steady-state cluster perturbation theory with an appropriate, consistent reference state. This reference state is obtained by the reduced many-body density matrix in the steady state obtained from a quantum master equation. The resulting hybrid method inherits beneficial aspects of steadystate cluster perturbation theory as well as from the quantum master equation.

We benchmarked this method on two experimentally realizable systems: a quantum diode and a triple quantum dot ring, which both feature negative differential conductance and interaction-induced current blocking effects. ME-CPT is able to improve the bare quantum master equation results by a correct inclusion of lead-induced level-broadening effects, and the correct noninteracting limit. In contrast to previous realizations of the steady-state cluster perturbation theory, ME-CPT is able to correctly predict interaction-induced current blocking effects. It is well known that the secular approximation (BMS-ME) is not applicable to quasidegenerate problems, which is corroborated by our results for the steady-state current. However, ME-CPT based on the BMS-ME density, is able to repair most of the shortcomings of BMS-ME. The results are very close to those obtained by ME-CPT based on the density of BM-ME, where the quasidegenerate states are treated consistently. 
The computational effort of ME-CPT beyond that of the bare quantum master equation scales with the number of significant entries in the reference state density matrix but is typically small. In the presented formulation, this method is flexible and fast and therefore well suited to study nanostructures, molecular junctions, or heterostructures also starting from an ab initio calculation [140].

\section{ACKNOWLEDGMENTS}

The authors acknowledge fruitful discussion with A. Rosch. This work was partly supported by the Austrian Science Fund (FWF) Grants No. P24081 and No. P26508 as well as SFBViCoM Projects No. F04103 and No. F04104 and NaWi Graz. M.N., G.D., and A.D. thank the Forschungszentrum Jülich, in particular the autumn school on correlated electrons, for hospitality and support.

\section{APPENDIX A: BORN-MARKOV AND PAULI MASTER EQUATION}

Here, we provide the detailed expressions for the coefficients in the BM-ME and BMS-ME of Eq. (6) and discuss the equations governing the time evolution into the steady state.

The Lamb-shift Hamiltonian is defined as $\hat{\mathcal{H}}^{\mathrm{LS}}=$ $\sum_{a b} \Lambda_{a b}|a\rangle\langle b|$, with

$$
\Lambda_{a b}=\frac{1}{2 i} \sum_{\alpha \beta} \sum_{c} \lambda_{\alpha \beta}\left(\omega_{b c}, \omega_{a c}\right)\left\langle c\left|\hat{S}_{\beta}\right| b\right\rangle\left\langle c\left|\hat{S}_{\alpha}\right| a\right\rangle^{*} .
$$

Note that $\left[\hat{\mathcal{H}}^{\mathrm{LS}}, \hat{\mathcal{H}}^{S}\right]_{-}=0$. In the secular approximation (BMS-ME), one can replace $\lambda_{\alpha \beta}\left(\omega_{b c}, \omega_{a c}\right) \rightarrow \lambda_{\alpha \beta}\left(\omega_{b}-\right.$ $\left.\omega_{c}\right) \delta_{\omega_{b}, \omega_{a}}$. The expressions for the BM-ME and BMS-ME (6) are valid if $\left[\hat{\mathcal{H}}^{E}, \hat{\rho}^{E}\right]_{-}=0$ and $\operatorname{tr}\left\{\hat{E}_{\alpha} \hat{\rho}^{E}\right\}=0$. The environment functions $\xi_{\alpha \beta}$ and $\lambda_{\alpha \beta}$ in Eqs. (A1) and (7) are determined by the time-dependent environment correlation functions

$$
C_{\alpha \beta}(\tau)=\operatorname{tr}\left\{\hat{E}_{\alpha}(\tau) \hat{E}_{\beta} \hat{\rho}_{E}\right\},
$$

where the Heisenberg time evolution in the environment operators is $\hat{E}_{\alpha}(\tau)=e^{+i \hat{\mathcal{H}}^{E} \tau} \hat{E}_{\alpha} e^{-i \hat{\mathcal{H}}^{E} \tau}$.

For the BM-ME, $\xi_{\alpha \beta}$ and $\lambda_{\alpha \beta}$ are given by a sum of complex Laplace transforms

$$
\begin{aligned}
& \xi_{\alpha \beta}\left(\omega_{1}, \omega_{2}\right)=\int_{0}^{\infty} d \tau C_{\alpha \beta}(\tau) e^{+i \omega_{1} \tau}+\int_{-\infty}^{0} d \tau C_{\alpha \beta}(\tau) e^{+i \omega_{2} \tau}, \\
& \lambda_{\alpha \beta}\left(\omega_{1}, \omega_{2}\right)=\int_{0}^{\infty} d \tau C_{\alpha \beta}(\tau) e^{+i \omega_{1} \tau}-\int_{-\infty}^{0} d \tau C_{\alpha \beta}(\tau) e^{+i \omega_{2} \tau},
\end{aligned}
$$

whereas for the BMS-ME $\left(\omega_{1}=\omega_{2}\right)$ the expressions simplify to the full even and odd Fourier transforms [77]

$$
\begin{gathered}
\xi_{\alpha \beta}(\omega)=\int_{-\infty}^{\infty} d \tau C_{\alpha \beta}(\tau) e^{+i \omega \tau} \\
\lambda_{\alpha \beta}(\omega)=\int_{-\infty}^{\infty} d \tau \operatorname{sign}(\tau) C_{\alpha \beta}(\tau) e^{+i \omega \tau} \\
=\frac{i}{\pi} \int_{-\infty}^{\infty} \mathcal{P} d \omega^{\prime} \frac{\xi_{\alpha \beta}\left(\omega^{\prime}\right)}{\omega-\omega^{\prime}} .
\end{gathered}
$$

The coupled equations for the real-time evolution of the components of the reduced system many-body density matrix $\rho_{a b}^{S}=\left\langle a\left|\hat{\rho}^{S}\right| b\right\rangle$ according to the BMS-ME read as

$$
\begin{aligned}
\dot{\rho}_{a b}^{S}(\tau)= & i\left(\omega_{b}-\omega_{a}\right) \rho_{a b}^{S}(\tau) \\
& +i \sum_{c}\left(\rho_{a c}^{S}(\tau) \Lambda_{c b}-\Lambda_{a c} \rho_{c b}^{S}(\tau)\right) \\
& +\sum_{c d}\left(\Xi_{a c, b d} \rho_{c d}^{S}(\tau)-\frac{1}{2} \Xi_{c d, c a} \rho_{d b}^{S}(\tau)\right. \\
& \left.-\frac{1}{2} \Xi_{c b, c d} \rho_{a d}^{S}(\tau)\right) .
\end{aligned}
$$

The equations simplify further for system Hamiltonians $\hat{\mathcal{H}}^{S}$ with nondegenerate eigenenergies $\omega_{a}$. Then, the diagonal components $\phi_{a}=\rho_{a a}^{S}$ decouple from the off-diagonals and one recovers the Pauli master equation for classical probabilities

$$
\dot{\phi}_{a}(\tau)=\sum_{c}\left(\Xi_{a c} \phi_{c}(\tau)-\Xi_{c a} \phi_{a}(\tau)\right),
$$

with simplified coefficients

$$
\Xi_{a b}:=\Xi_{a b, a b}=\sum_{\alpha \beta} \xi_{\alpha \beta}\left(\omega_{b}-\omega_{a}\right)\left\langle a\left|\hat{S}_{\beta}\right| b\right\rangle\left\langle a\left|\hat{S}_{\alpha}\right| b\right\rangle^{*} .
$$

In this case, the dynamics of the decoupled off-diagonal terms $(a \neq b)$ is given by

$$
\begin{aligned}
\dot{\rho}_{a b}^{S}(\tau)= & {\left[i\left(\omega_{b}+\Lambda_{b}-\omega_{a}-\Lambda_{a}\right)\right.} \\
& \left.-\frac{1}{2} \sum_{c}\left(\Xi_{c a}+\Xi_{c b}\right)\right] \rho_{a b}^{S}(\tau),
\end{aligned}
$$

where the simplified Lamb-shift terms are

$$
\Lambda_{a}:=\Lambda_{a a}=\frac{1}{2 i} \sum_{\alpha \beta} \sum_{c} \lambda_{\alpha \beta}\left(\omega_{a}-\omega_{c}\right)\left\langle c\left|\hat{S}_{\beta}\right| a\right\rangle\left\langle c\left|\hat{S}_{\alpha}\right| a\right\rangle^{*}
$$

\section{APPENDIX B: HERMITIAN TENSOR PRODUCT FORM OF THE COUPLING HAMILTONIAN}

For the BMS-ME (see Sec. III B), it is necessary to bring the fermionic system-environment coupling Hamiltonian (1c) to a Hermitian tensor product form, which requires $\left[\hat{S}_{\alpha}, \hat{E}_{\alpha}\right]_{-}=0$. For the fermionic operators in Eq. (1c) we, however, have $\left[f_{i \sigma}^{\dagger}, c_{\lambda k \sigma}\right]_{-}=2 f_{i \sigma}^{\dagger} c_{\lambda k \sigma}$. A solution is provided in Ref. [78] by performing a Jordan-Wigner transformation [141] on the system and environment operators

$$
\begin{aligned}
f_{i \sigma} & =\prod_{\sigma}\left(\xi_{1}^{z} \otimes \ldots \otimes \xi_{i-1}^{z} \xi_{i}^{-} \mathbb{1}_{i+1} \otimes \ldots \otimes \mathbb{1}_{L_{S}}\right)_{S, \sigma} \\
& \otimes \prod_{\lambda}\left(1_{1} \otimes \ldots \otimes \mathbb{1}_{L_{E}}\right)_{E, \lambda \sigma}, \\
c_{\lambda j \sigma} & =\prod_{\sigma}\left(\xi_{1}^{z} \otimes \ldots \otimes \xi_{L_{S}}^{z}\right)_{S, \sigma} \\
& \otimes \prod_{\lambda}\left(\eta_{1}^{z} \otimes \ldots \otimes \eta_{j-1}^{z} \eta_{j}^{-} 1_{j+1} \otimes \ldots \otimes \mathbb{1}_{L_{E}}\right)_{E, \lambda \sigma},
\end{aligned}
$$


where $\xi_{i}$ and $\eta_{j}$ denote local spin- $\frac{1}{2}$ degrees of freedom at the system and environment sites, respectively, and the overall ordering of operators is important. $L_{S} / L_{E}$ denote the size of the system/environment. Reordering Eq. (1c) we find
$\hat{\mathcal{H}}_{\lambda}^{S E}=\sum_{i j \sigma} t_{\lambda i j \sigma}^{\prime} f_{i \sigma}^{\dagger} c_{\lambda j \sigma}-t_{\lambda i j \sigma}^{\prime *} f_{i \sigma} c_{\lambda j \sigma}^{\dagger}$, where the minus sign arises due to the fermionic anticommutator. Plugging in the Jordan-Wigner transformed operators leads to

$$
\begin{aligned}
\hat{\mathcal{H}}_{\lambda}^{S E}= & \sum_{i j \sigma}\left(t_{\lambda i j \sigma}^{\prime}\left[\xi_{i}^{+} \otimes\left[-\xi_{i+1}^{z} \otimes \ldots \otimes \xi_{L_{S}}^{z} \otimes \eta_{1}^{z} \otimes \ldots \otimes \eta_{j-1}^{z}\right] \otimes \eta_{j}^{-}\right]_{\sigma \lambda}+t_{\lambda i j \sigma}^{\prime *}\right. \\
& \left.\times\left[\xi_{i}^{-} \otimes\left[-\xi_{i+1}^{z} \otimes \ldots \otimes \xi_{L_{S}}^{z} \otimes \eta_{1}^{z} \otimes \ldots \otimes \eta_{j-1}^{z}\right] \otimes \eta_{j}^{+}\right]_{\sigma \lambda}\right) \\
= & \sum_{i}\left(\bar{f}_{i}^{\dagger} \otimes \bar{c}_{i}+\bar{f}_{i} \otimes \bar{c}_{i}^{\dagger}\right)
\end{aligned}
$$

where in the last line we have defined new operators

$$
\begin{aligned}
\bar{f}_{i \sigma} & =\left[\xi_{i}^{-} \otimes\left[-\xi_{i+1}^{z} \otimes \ldots \otimes \xi_{L_{S}}^{z}\right]\right]_{\sigma}, \\
\bar{f}_{i \sigma}^{\dagger} & =\left[\left[-\xi_{i+1}^{z} \otimes \ldots \otimes \xi_{L_{S}}^{z}\right] \otimes \xi_{i}^{+}\right]_{\sigma}, \\
\bar{c}_{\lambda i \sigma} & =\sum_{j} t_{\lambda i j \sigma}^{\prime}\left[\left[\eta_{1}^{z} \otimes \ldots \otimes \eta_{j-1}^{z}\right] \otimes \eta_{j}^{-}\right]_{\lambda \sigma}, \\
\bar{c}_{\lambda i \sigma}^{\dagger} & =\sum_{j} t_{\lambda i j \sigma}^{\prime *}\left[\eta_{j}^{+} \otimes\left[\eta_{1}^{z} \otimes \ldots \otimes \eta_{j-1}^{z}\right]\right]_{\lambda \sigma} .
\end{aligned}
$$

Note that the phase operator $\hat{P}_{i(j \lambda) \sigma}=\left[-\xi_{i+1}^{z} \otimes \ldots \otimes \xi_{L_{S}}^{z}\right.$

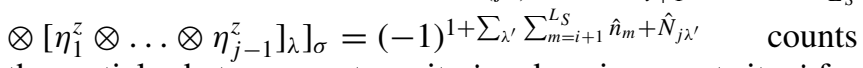
the particles between system site $i$ and environment site $j$ for spin $\sigma$ depending on the ordering of the environments $\lambda$. It is straightforward to show that the bar operators fulfill fermionic anticommutation rules. Furthermore, $\left[\bar{f}_{i \sigma}, \bar{c}_{\lambda i \sigma}\right]_{-}=0$, which allows us to write the coupling Hamiltonian in a tensor product form. Note that in general $\left[\bar{f}_{i \sigma}, \bar{c}_{\lambda^{\prime} j \sigma}\right]_{-} \neq 0$ for $i \neq j$ which is, however, not relevant for the coupling Hamiltonian where only the same $i$ couple.

The new operators in Hermitian form are given in Eq. (5) by replacing $c \rightarrow \bar{c}$ and $f \rightarrow \bar{f}$. Next we show, by examining the BMS-ME, that in most cases the additional phase operator in $\bar{c}$ drops out of the calculations and we are even allowed to use the original $f$ and $c$ operators instead of the barred ones. The operators $\bar{c}$ only enter the equations in the environment correlation functions $C_{\alpha \beta}(\tau)$ as defined in Eq. (A2). Plugging in the barred operators we obtain for normal systems which preserve particle number

$$
C_{\alpha \beta}(\tau) \propto \operatorname{tr}\left\{e^{+i \hat{\mathcal{H}}^{E} \tau} f_{\lambda j \sigma}^{\dagger} e^{-i \hat{\mathcal{H}}^{E} \tau} \hat{P}_{i(j \lambda) \sigma}^{2} c_{\lambda j \sigma} \hat{\rho}^{E}\right\},
$$

with $\hat{P}_{i j}^{2}=11$, where we required that $\left[\hat{\mathcal{H}}^{E}, \hat{P}_{i j}\right]_{-}=0$. The dropping out of the phase operators implies that for normal systems where the disconnected environments conserve particle number we can omit the Jordan-Wigner transformation and do all calculations as is with the original environment creation/annihilation operators in Hermitian form.

\section{APPENDIX C: BATH CORRELATION FUNCTIONS}

In the wide band limit, analytical expressions for the bath correlation functions are available in Ref. [38]. For arbitrary environment DOS, explicit evaluation of the environment correlation functions becomes convenient for Hermitian cou- plings [Eq. (5)] as outlined in Appendix B [77]. Essentially, the environment functions can all be obtained via integrals of the environment DOS $\rho(\omega)$. Care has to be taken when going to very low temperatures and solving the integrals with finite precision arithmetic to avoid underflow errors.

The time-dependent environment correlation functions $C_{\alpha \beta}(\tau)$ [Eq. (A2)] become

$$
\begin{aligned}
C_{11}(\tau)= & C_{22}(\tau)=\frac{1}{4 \pi} \sum_{\lambda \sigma} \int_{-\infty}^{\infty} d v \Gamma_{\lambda \sigma}(\nu) \\
& \times\left(e^{-i v \tau}+2 i p_{\mathrm{FD}}\left(\nu, T_{\lambda}, \mu_{\lambda}\right) \sin (\nu \tau)\right), \\
C_{12}(\tau)= & -C_{21}(\tau)=\frac{i}{4 \pi} \sum_{\lambda \sigma} \int_{-\infty}^{\infty} d \nu \Gamma_{\lambda \sigma}(\nu) \\
& \times\left(-e^{-i \nu \tau}+2 p_{\mathrm{FD}}\left(\nu, T_{\lambda}, \mu_{\lambda}\right) \cos (\nu \tau)\right),
\end{aligned}
$$

where $C_{\alpha \beta}(\tau)=C_{\beta \alpha}^{\dagger}(-\tau)$ and the coefficient

$$
\Gamma_{\lambda \sigma}(v)=2 \pi\left|t_{\lambda \sigma}^{\prime}\right|^{2} \sum_{k} \delta\left(v-\omega_{\lambda k \sigma}\right)
$$

is proportional to the lead DOS.

For the BMS-ME, the respective full even Fourier transforms $\xi_{\alpha \beta}(\omega)$ [Eq. (A5)], we find

$$
\begin{aligned}
\xi_{11}(\omega)= & \xi_{22}(\omega)=\frac{1}{2} \sum_{\lambda \sigma} \Gamma_{\lambda \sigma}(-\omega) p_{\mathrm{FD}}\left(-\omega, \beta_{\lambda}, \mu_{\lambda}\right) \\
& +\Gamma_{\lambda \sigma}(\omega) \bar{p}_{\mathrm{FD}}\left(\omega, T_{\lambda}, \mu_{\lambda}\right), \\
\xi_{12}(\omega)= & -\xi_{21}(\omega)=\frac{i}{2} \sum_{\lambda \sigma} \Gamma_{\lambda \sigma}(-\omega) p_{\mathrm{FD}}\left(-\omega, \beta_{\lambda}, \mu_{\lambda}\right) \\
& -\Gamma_{\lambda \sigma}(\omega) \bar{p}_{\mathrm{FD}}\left(\omega, T_{\lambda}, \mu_{\lambda}\right),
\end{aligned}
$$

where $\bar{p}_{\mathrm{FD}}(\omega, T, \mu)=1-p_{\mathrm{FD}}(\omega, T, \mu)$.

The odd Fourier transforms $\lambda_{\alpha \beta}(\omega)$ [Eq. (A6)] are given by

$$
\begin{aligned}
\lambda_{11}(\omega)= & \lambda_{22}(\omega)=\frac{i}{2 \pi} \sum_{\lambda \sigma} \int_{-\infty}^{\infty} \mathcal{P} d \nu \Gamma_{\lambda \sigma}(v) \\
& \times\left(\frac{p_{\mathrm{FD}}\left(v, \beta_{\lambda}, \mu_{\lambda}\right)}{v+\omega}-\frac{\bar{p}_{\mathrm{FD}}\left(\nu, \beta_{\lambda}, \mu_{\lambda}\right)}{v-\omega}\right), \\
\lambda_{12}(\omega)= & -\lambda_{21}(\omega)=-\frac{1}{2 \pi} \sum_{\lambda \sigma} \int_{-\infty}^{\infty} \mathcal{P} d \nu \Gamma_{\lambda \sigma}(v) \\
& \times\left(\frac{p_{\mathrm{FD}}\left(v, \beta_{\lambda}, \mu_{\lambda}\right)}{v+\omega}+\frac{\bar{p}_{\mathrm{FD}}\left(\nu, \beta_{\lambda}, \mu_{\lambda}\right)}{v-\omega}\right) .
\end{aligned}
$$




\section{APPENDIX D: EVALUATION OF STEADY-STATE OBSERVABLES}

\section{Steady-state cluster perturbation theory}

Within ME-CPT, single-particle observables are available by integration of $\widetilde{G}(\omega)$ [Eq. (4)]. It is easy to show that the single-particle density matrix $\kappa_{i j \sigma}=\frac{\delta_{i j}}{2}-\frac{i}{2} \int_{-\infty}^{\infty} \frac{d \omega}{2 \pi} G_{i j \sigma}^{K}(\omega)$ can be expressed in terms of the retarded CPT Green's function

$$
\begin{aligned}
\kappa_{i j \sigma}= & \frac{\delta_{i j}}{2}-\frac{i}{2} \int_{-\infty}^{\infty} \frac{d \omega}{2 \pi}\left(G_{i n \sigma}^{R}(\omega) P_{n j \sigma}(\omega)-P_{i n \sigma}(\omega)\left[G_{j n \sigma}^{R}(\omega)\right]^{*}\right. \\
& \left.+G_{i n \sigma}^{R}(\omega)\left(\left[P_{\sigma}(\omega), M_{\sigma}\right]_{-}\right)_{n m}\left[G_{j m \sigma}^{R}(\omega)\right]^{*}\right),
\end{aligned}
$$

where $M_{\sigma}$ is the intercluster perturbation defined in Eq. (4). Here, we use the Einstein summation convention, the last line holds within CPT, and $P_{i j \sigma}(\omega)=\delta_{i j}\left[1-2 p_{\mathrm{FD}}\left(\omega, T_{i}, \mu_{i \sigma}\right)\right]$.

From the real part of the single-particle density matrix we read off the site occupation $\left\langle n_{i}\right\rangle=\sum_{\sigma} \kappa_{i i \sigma}$ the spinresolved occupations $\left\langle n_{i \sigma}\right\rangle=\kappa_{i i \sigma}$ and the magnetization $\left\langle m_{i}\right\rangle=\frac{1}{2}\left(\kappa_{i i \uparrow}-\kappa_{i i \downarrow}\right)$.

The current $\left\langle j_{\langle i j\rangle}\right\rangle$ between nearest-neighbor sites $\langle i j\rangle$ is related to the imaginary part of $\kappa_{i j \sigma}$ and reads as in symmetrized form

$$
\left\langle j_{\langle i j\rangle}\right\rangle=\frac{e}{2 \hbar}\left(h_{i j \sigma} \kappa_{i j \sigma}-h_{j i \sigma} \kappa_{j i \sigma}\right),
$$

which is of Meir-Wingreen form [81] and $h_{i j \sigma}$ is the singleparticle Hamiltonian.

Equivalently, the transmission current between two environments $\lambda=1,2$ can be evaluated in the Landauer-Büttiker form $[54,64,97]$

$$
\left\langle j_{1 / 2}\right\rangle=\frac{e}{\hbar} \int_{-\infty}^{\infty} \frac{d \omega}{2 \pi} W(\omega) \operatorname{tr}\{\mathcal{T}(\omega)\},
$$

with the transport window

$$
W(\omega)=p_{\mathrm{FD}}\left(\omega, T_{1}, \mu_{1}\right)-p_{\mathrm{FD}}\left(\omega, T_{2}, \mu_{2}\right),
$$

and where the transmission function

$$
\mathcal{T}(\omega)=G^{R}(\omega) \Gamma_{1}(\omega)\left[G^{R}(\omega)\right]^{\dagger} \Gamma_{2}(\omega)
$$

is given in terms of $G^{R}(\omega)=\left(\left[g^{R}(\omega)\right]^{-1}-\left(\widetilde{\Sigma}_{1}+\widetilde{\Sigma}_{2}\right)\right)^{-1}$ with the lead broadening functions of lead $\lambda$ projected onto the system sites $i, j$ is $\widetilde{\Sigma}_{\lambda i j}=M_{i \lambda} g_{\lambda \lambda}^{R} M_{\lambda j}$ and $\Gamma_{\lambda}=-2 \operatorname{Im}\left(\widetilde{\Sigma}_{\lambda}\right)$ [compare also Eq. (C1)].

\section{Born-Markov master equation}

Within the ME, basic single-particle observables are available in terms of the reduced system many-body density matrix $\hat{\rho}^{S}$. The single-particle density matrix $\kappa$ reads as

$$
\kappa_{i j \sigma}=\operatorname{tr}\left(f_{i \sigma}^{\dagger} f_{j \sigma} \hat{\rho}^{S}\right)=\sum_{a b}\left\langle b\left|f_{i \sigma}^{\dagger} f_{j \sigma}\right| a\right\rangle \rho_{a b}^{S},
$$

where $a$ and $b$ denote eigenstates of the system Hamiltonian. Note that within the BM-ME/BMS-ME $\kappa_{i j \sigma}$ is purely real and therefore does predict zero current.

However, an expression for the current to reservoir $\lambda$ can be found by making use of the operator of total system charge $\hat{Q}$ and total system particle number $\hat{N}$, where $q$ denotes the charge of one carrier

$$
\sum_{\lambda} j^{\lambda}(\tau)=\frac{d}{d \tau}\langle\hat{Q}(\tau)\rangle=q \operatorname{tr}\left(\hat{N} \dot{\hat{\rho}}^{S}(\tau)\right) .
$$

Taking $\dot{\hat{\rho}}^{S}(\tau)$ from the ME, we obtain

$$
j^{\lambda}=q \sum_{a b c}\left[\left(n_{c}-\frac{1}{2} n_{b}-\frac{1}{2} n_{a}\right) \Xi_{c a, c b}^{\lambda}\right] \rho_{a b}^{S},
$$

and for nondegenerate systems, in the Pauli limit we find from the BMS-ME

$$
j_{\text {non-deg }}^{\lambda}=q \sum_{a b}\left(n_{a}-n_{b}\right) \Xi_{a b}^{\lambda} \phi_{b} .
$$

[1] G. Cuniberti, G. Fagas, and K. Richter, Introducing Molecular Electronics (Springer, Berlin, 2005).

[2] A. Nitzan and M. A. Ratner, Science 300, 1384 (2003).

[3] N. Agrait, A. L. Yeyati, and J. M. van Ruitenbeek, Phys. Rep. 377, 81 (2003).

[4] J. C. Cuevas and E. Scheer, Molecular Electronics: An Introduction to Theory and Experiment, 1st ed. (World Scientific, Singapore, 2010).

[5] Y. V. Nazarov and Y. M. Blanter, Quantum Transport: Introduction to Nanoscience (Cambridge University Press, New York, 2009).

[6] M. D. Ventra, Electrical Transport in Nanoscale Systems (Cambridge University Press, New York, 2008).

[7] D. K. Ferry, S. M. Goodnick, and J. Bird, Transport in Nanostructures, 2nd ed. (Cambridge University Press, Cambridge, UK, 2009).

[8] L. Grill, M. Dyer, L. Lafferentz, M. Persson, M. V. Peters, and S. Hecht, Nat. Nanotechnol. 2, 687 (2007).

[9] J. Park, A. N. Pasupathy, J. I. Goldsmith, C. Chang, Y. Yaish, J. R. Petta, M. Rinkoski, J. P. Sethna, H. D. Abruna, P. L. McEuen et al., Nature (London) 417, 722 (2002).
[10] W. Liang, M. P. Shores, M. Bockrath, J. R. Long, and H. Park, Nature (London) 417, 725 (2002).

[11] S. Kubatkin, A. Danilov, M. Hjort, J. Cornil, J.-L. Bredas, N. Stuhr-Hansen, P. Hedegard, and T. Bjornholm, Nature (London) 425, 698 (2003).

[12] L. H. Yu, Z. K. Keane, J. W. Ciszek, L. Cheng, J. M. Tour, T. Baruah, M. R. Pederson, and D. Natelson, Phys. Rev. Lett. 95, 256803 (2005).

[13] D.-H. Chae, J. F. Berry, S. Jung, F. A. Cotton, C. A. Murillo, and Z. Yao, Nano Lett. 6, 165 (2006).

[14] M. Poot, E. Osorio, K. O’Neill, J. M. Thijssen, D. Vanmaekelbergh, C. A. van Walree, L. W. Jenneskens, and H. S. J. van der Zant, Nano Lett. 6, 1031 (2006).

[15] H. B. Heersche, Z. de Groot, J. A. Folk, H. S. J. van der Zant, C. Romeike, M. R. Wegewijs, L. Zobbi, D. Barreca, E. Tondello, and A. Cornia, Phys. Rev. Lett. 96, 206801 (2006).

[16] E. Osorio, K. O’Neill, N. Stuhr-Hansen, O. Nielsen, T. Bjrnholm, and H. van der Zant, Adv. Mater. 19, 281 (2007).

[17] A. Danilov, S. Kubatkin, S. Kafanov, P. Hedegrd, N. StuhrHansen, K. Moth-Poulsen, and T. Bjrnholm, Nano Lett. 8, 1 (2008). 
[18] R. H. M. Smit, Y. Noat, C. Untiedt, N. D. Lang, M. C. van Hemert, and J. M. van Ruitenbeek, Nature (London) 419, 906 (2002).

[19] A. R. Champagne, A. N. Pasupathy, and D. C. Ralph, Nano Lett. 5, 305 (2005).

[20] E. Lörtscher, H. B. Weber, and H. Riel, Phys. Rev. Lett. 98, 176807 (2007).

[21] M. Kiguchi, O. Tal, S. Wohlthat, F. Pauly, M. Krieger, D. Djukic, J. C. Cuevas, and J. M. van Ruitenbeek, Phys. Rev. Lett. 101, 046801 (2008).

[22] D. I. Gittins, D. Bethell, D. J. Schiffrin, and R. J. Nichols, Nature (London) 408, 67 (2000).

[23] Xiao, Xu, and N. J. Tao, Nano Lett. 4, 267 (2004).

[24] J. Repp, G. Meyer, S. M. Stojković, A. Gourdon, and C. Joachim, Phys. Rev. Lett. 94, 026803 (2005).

[25] L. Venkataraman, J. E. Klare, C. Nuckolls, M. S. Hybertsen, and M. L. Steigerwald, Nature (London) 442, 904 (2006).

[26] M. Koch, F. Ample, C. Joachim, and L. Grill, Nat. Nanotechnol. 7, 713 (2012).

[27] M. A. Reed, C. Zhou, C. J. Muller, T. P. Burgin, and J. M. Tour, Science 278, 252 (1997).

[28] M. C. Rogge and R. J. Haug, Phys. Rev. B 78, 153310 (2008).

[29] L. Gaudreau, A. Kam, G. Granger, S. A. Studenikin, P. Zawadzki, and A. S. Sachrajda, Appl. Phys. Lett. 95, 193101 (2009).

[30] F. Molitor, S. Drscher, J. Gttinger, A. Jacobsen, C. Stampfer, T. Ihn, and K. Ensslin, Appl. Phys. Lett. 94, 222107 (2009).

[31] J. Eroms and D. Weiss, New J. Phys. 11, 095021 (2009).

[32] N. Tombros, C. Jozsa, M. Popinciuc, H. T. Jonkman, and B. J. van Wees, Nature (London) 448, 571 (2007).

[33] L. Gaudreau, S. A. Studenikin, A. S. Sachrajda, P. Zawadzki, A. Kam, J. Lapointe, M. Korkusinski, and P. Hawrylak, Phys. Rev. Lett. 97, 036807 (2006).

[34] L. Gaudreau, A. S. Sachrajda, S. Studenikin, A. Kam, F. Delgado, Y. P. Shim, M. Korkusinski, and P. Hawrylak, Phys. Rev. B 80, 075415 (2009).

[35] G. Austing, C. Payette, G. Yu, J. Gupta, G. Aers, S. Nair, S. Amaha, and S. Tarucha, Jpn. J. Appl. Phys. 49, 04DJ03 (2010).

[36] L. Kouwenhoven, C. Marcus, P. McEuen, S. Tarucha, R. Westervelt, and N. Wingreen, Kluwer Series, Proc. NATO Adv. Study Inst. Mesoscopic Electron Transport E345, 105 (1997).

[37] B. William, Electronic and Optical Properties of Conjugated Polymers (Oxford University Press, Oxford, UK, 2005).

[38] G. Begemann, D. Darau, A. Donarini, and M. Grifoni, Phys. Rev. B 77, 201406 (2008).

[39] D. Darau, G. Begemann, A. Donarini, and M. Grifoni, Phys. Rev. B 79, 235404 (2009).

[40] D. M. Cardamone, C. A. Stafford, and S. Mazumdar, Nano Lett. 6, 2422 (2006).

[41] A. Gagliardi, G. C. Solomon, A. Pecchia, T. Frauenheim, A. Di Carlo, N. S. Hush, and J. R. Reimers, Phys. Rev. B 75, 174306 (2007).

[42] Z. Qian, R. Li, X. Zhao, S. Hou, and S. Sanvito, Phys. Rev. B 78, 113301 (2008).

[43] S.-H. Ke, W. Yang, and H. U. Baranger, Nano Lett. 8, 3257 (2008).

[44] A. Donarini, G. Begemann, and M. Grifoni, Nano Lett. 9, 2897 (2009).

[45] A. Donarini, G. Begemann, and M. Grifoni, Phys. Rev. B 82, 125451 (2010).
[46] N. Roch, S. Florens, V. Bouchiat, W. Wernsdorfer, and F. Balestro, Nature (London) 453, 633 (2008).

[47] D. Lobaskin and S. Kehrein, Phys. Rev. B 71, 193303 (2005).

[48] A. C. Hewson, The Kondo Problem to Heavy Fermions (Cambridge University Press, Cambridge, UK, 1997).

[49] D. Goldhaber-Gordon, J. Göres, M. A. Kastner, H. Shtrikman, D. Mahalu, and U. Meirav, Phys. Rev. Lett. 81, 5225 (1998).

[50] S. De Franceschi, R. Hanson, W. G. van der Wiel, J. M. Elzerman, J. J. Wijpkema, T. Fujisawa, S. Tarucha, and L. P. Kouwenhoven, Phys. Rev. Lett. 89, 156801 (2002).

[51] R. Leturcq, L. Schmid, K. Ensslin, Y. Meir, D. C. Driscoll, and A. C. Gossard, Phys. Rev. Lett. 95, 126603 (2005).

[52] A. V. Kretinin, H. Shtrikman, D. Goldhaber-Gordon, M. Hanl, A. Weichselbaum, J. von Delft, T. Costi, and D. Mahalu, Phys. Rev. B 84, 245316 (2011).

[53] A. V. Kretinin, H. Shtrikman, and D. Mahalu, Phys. Rev. B 85, 201301 (2012).

[54] H. Haug and A. Jauho, Quantum Kinetics in Transport and Optics of Semiconductors, 2nd ed. (Springer, Berlin, 1996).

[55] P. Myöhänen, A. Stan, G. Stefanucci, and R. van Leeuwen, Phys. Rev. B 80, 115107 (2009).

[56] J.-S. Wang, B. Agarwalla, H. Li, and J. Thingna, Front. Phys. 9, 673 (2013).

[57] T. Kubis and P. Vogl, J. Comput. Electron. 6, 183 (2006).

[58] P. Delaney and J. C. Greer, Phys. Rev. Lett. 93, 036805 (2004).

[59] M. Strange, I. S. Kristensen, K. S. Thygesen, and K. W. Jacobsen, J. Chem. Phys. 128, 114714 (2008).

[60] J. Chen, K. S. Thygesen, and K. W. Jacobsen, Phys. Rev. B 85, 155140 (2012).

[61] M. Strange and K. S. Thygesen, Beilstein J. Nanotechnol. 2, 746 (2011).

[62] D. A. Ryndyk, R. Gutiérrez, B. Song, and G. Cuniberti, in Energy Transfer Dynamics in Biomaterial Systems, edited by I. Burghardt, V. May, D. A. Micha, and E. R. Bittner (Springer, Berlin, 2009), Vol. 93, pp. 213-335.

[63] K. Richter, Semiclassical Theory of Mesoscopic Quantum Systems (Springer, Berlin, 1999).

[64] S. Datta, Quantum Transport: Atom to Transistor, 2nd ed. (Cambridge University Press, Cambridge, UK, 2005).

[65] S. Andergassen, V. Meden, H. Schoeller, J. Splettstoesser, and M. R. Wegewijs, Nanotechnology 21, 272001 (2010).

[66] H. Schoeller, Eur. Phys. J. Spec. Top. 168, 179 (2009).

[67] A. Rosch, J. Paaske, J. Kroha, and P. Wölfle, J. Phys. Soc. Jpn. 74, 118 (2005).

[68] A. Hackl and S. Kehrein, J. Phys.: Condens. Matter 21, 015601 (2009).

[69] J. Eckel, F. Heidrich-Meisner, S. G. Jakobs, M. Thorwart, M. Pletyukhov, and R. Egger, New. J. Phys. 12, 043042 (2010).

[70] J. F. Rentrop, S. G. Jakobs, and V. Meden, J. Phys. A: Math. Theor. 48, 145002 (2015).

[71] H. Aoki, N. Tsuji, M. Eckstein, M. Kollar, T. Oka, and P. Werner, Rev. Mod. Phys. 86, 779 (2014).

[72] U. Schollwöck, Ann. Phys. (NY) 326, 96 (2011).

[73] F. B. Anders, Phys. Rev. Lett. 101, 066804 (2008).

[74] H.-P. Breuer and F. Petruccione, The Theory of Open Quantum Systems (Oxford University Press, Oxford, UK, 2002).

[75] H. J. Carmichael, An Open Systems Approach to Quantum Optics: Lectures Presented at the Universite Libre De Bruxelles October 28 to November 4, 1991 (Lecture Notes in Physics New Series M) (Springer, Berlin, 1993). 
[76] H. J. Carmichael, Statistical Methods in Quantum Optics 1: Master Equations and Fokker-Planck Equations (Springer, Berlin, 2010).

[77] G. Schaller, Non-Equilibrium Master Equations (Lecture Notes TU Berlin, Berlin, 2014).

[78] G. Schaller, Open Quantum Systems Far from Equilibrium (Springer, New York, 2014).

[79] C. Cohen-Tannoudji, J. Dupont-Roc, and G. Grynberg, AtomPhoton Interactions: Basic Processes and Applications (Wiley, New York, 1998).

[80] J. Jin, J. Li, Y. Liu, X.-Q. Li, and Y. Yan, J. Chem. Phys. 140, 244111 (2014).

[81] Y. Meir and N. S. Wingreen, Phys. Rev. Lett. 68, 2512 (1992).

[82] H. Schoeller and G. Schön, Phys. Rev. B 50, 18436 (1994).

[83] M. Eckstein, A. Hackl, S. Kehrein, M. Kollar, M. Moeckel, P. Werner, and F. Wolf, Eur. Phys. J. Spec. Top. 180, 217 (2010).

[84] R. Gezzi, T. Pruschke, and V. Meden, Phys. Rev. B 75, 045324 (2007).

[85] S. G. Jakobs, V. Meden, and H. Schoeller, Phys. Rev. Lett. 99, 150603 (2007).

[86] J. K. Freericks, V. M. Turkowski, and V. Zlatić, Phys. Rev. Lett. 97, 266408 (2006)

[87] M. Potthoff, M. Aichhorn, and C. Dahnken, Phys. Rev. Lett. 91, 206402 (2003).

[88] C. Gros and R. Valenti, Phys. Rev. B 48, 418 (1993).

[89] D. Sénéchal, D. Perez, and M. Pioro-Ladriére, Phys. Rev. Lett. 84, 522 (2000).

[90] M. Balzer and M. Potthoff, Phys. Rev. B 83, 195132 (2011).

[91] F. Hofmann, M. Eckstein, E. Arrigoni, and M. Potthoff, Phys. Rev. B 88, 165124 (2013).

[92] M. Knap, W. von der Linden, and E. Arrigoni, Phys. Rev. B 84, 115145 (2011).

[93] M. Nuss, E. Arrigoni, M. Aichhorn, and W. von der Linden, Phys. Rev. B 85, 235107 (2012).

[94] M. Nuss, C. Heil, M. Ganahl, M. Knap, H. G. Evertz, E. Arrigoni, and W. von der Linden, Phys. Rev. B 86, 245119 (2012).

[95] M. Nuss, E. Arrigoni, and W. von der Linden, AIP Conf. Proc. 1485, 302 (2012).

[96] M. Nuss, W. von der Linden, and E. Arrigoni, Phys. Rev. B 89, 155139 (2014).

[97] M. Knap, E. Arrigoni, and W. von der Linden, Phys. Rev. B 88, 054301 (2013).

[98] J. Jin, M. W.-Y. Tu, W.-M. Zhang, and Y. Yan, New J. Phys. 12, 083013 (2010).

[99] A. A. Dzhioev and D. S. Kosov, J. Phys.: Condens. Matter 24, 225304 (2012)

[100] W.-M. Zhang, P.-Y. Lo, H.-N. Xiong, M. W.-Y. Tu, and F. Nori, Phys. Rev. Lett. 109, 170402 (2012).

[101] A. A. Dzhioev and D. S. Kosov, J. Phys. A: Math. Theor. 48, 015004 (2015).

[102] E. Arrigoni, M. Knap, and W. von der Linden, Phys. Rev. Lett. 110, 086403 (2013).

[103] A. Dorda, M. Nuss, W. von der Linden, and E. Arrigoni, Phys. Rev. B 89, 165105 (2014).

[104] A. Georges, G. Kotliar, W. Krauth, and M. J. Rozenberg, Rev. Mod. Phys. 68, 13 (1996).

[105] W. Metzner and D. Vollhardt, Phys. Rev. Lett. 62, 324 (1989).

[106] P. Schmidt and H. Monien, arXiv:cond-mat/0202046.
[107] J. W. Negele and H. Orland, Quantum Many-Particle Systems (Westview Press, Boulder, CO, 1998).

[108] Throughout this paper we use $\hbar=k_{B} \equiv 1$.

[109] J. Schwinger, J. Math. Phys. 2, 407 (1961).

[110] R. P. Feynman and F. L. Vernon Jr., Ann. Phys. (NY) 24, 118 (1963).

[111] L. Keldysh, Zh. Eksp. Teor. Fiz. 47, 1515 (1965).

[112] C. H. Fleming and N. I. Cummings, Phys. Rev. E 83, 031117 (2011).

[113] T. Mori and S. Miyashita, J. Phys. Soc. Jpn. 77, 124005 (2008).

[114] $[\hat{a}, \hat{b}]_{ \pm}=\hat{a} \hat{b} \pm \hat{b} \hat{a}$ denotes the anticommutator and commutator, respectively.

[115] D. Sénéchal, arXiv:0806.2690.

[116] J. Rammer and H. Smith, Rev. Mod. Phys. 58, 323 (1986).

[117] E. N. Economou, Green's Functions in Quantum Physics, 3rd ed. (Springer, Berlin, 2010).

[118] Z. Bai, J. Demmel, J. Dongarra, A. Ruhe, and H. van der Vorst, Templates for the Solution of Algebraic Eigenvalue Problems: A Practical Guide (Software, Environments and Tools) (Society for Industrial and Applied Mathematics, Philadelphia, 1987).

[119] W. H. Press, S. A. Teukolsky, W. T. Vetterling, and B. P. Flannery, Numerical Recipes: The Art of Scientific Computing, 3rd ed. (Cambridge University Press, Cambridge, UK, 2007).

[120] Assuming that the interacting part is small enough to be treated exactly, otherwise it has to be decomposed into subpartitions again.

[121] We denote the largest energy scale or parameter of $\hat{\mathcal{H}}$ as $|\hat{\mathcal{H}}|$.

[122] R. S. Whitney, J. Phys. A: Math. Theor. 41, 175304 (2008).

[123] Y. Yan, F. Shuang, R. Xu, J. Cheng, X.-Q. Li, C. Yang, and H. Zhang, J. Chem. Phys. 113, 2068 (2000).

[124] P. W. Anderson, Phys. Rev. 124, 41 (1961).

[125] M. I. Katsnelson, V. Y. Irkhin, L. Chioncel, A. I. Lichtenstein, and R. A. de Groot, Rev. Mod. Phys. 80, 315 (2008).

[126] I. Bloch, J. Dalibard, and W. Zwerger, Rev. Mod. Phys. 80, 885 (2008).

[127] J. Hubbard, Proc. R. Soc. London, Ser. A 276, 238 (1963).

[128] A. Vidan, R. M. Westervelt, M. Stopa, M. Hanson, and A. C. Gossard, Appl. Phys. Lett. 85, 3602 (2004).

[129] F. Delgado, Y.-P. Shim, M. Korkusinski, L. Gaudreau, S. A. Studenikin, A. S. Sachrajda, and P. Hawrylak, Phys. Rev. Lett. 101, 226810 (2008).

[130] W. Gong, Y. Zheng, and T. Lü. Appl. Phys. Lett. 92, 042104 (2008).

[131] T. Kostyrko and B. R. Bułka, Phys. Rev. B 79, 075310 (2009).

[132] Y.-P. Shim, F. Delgado, and P. Hawrylak, Phys. Rev. B 80, 115305 (2009).

[133] C. Pöltl, C. Emary, and T. Brandes, Phys. Rev. B 80, 115313 (2009).

[134] C. Emary, Phys. Rev. B 76, 245319 (2007).

[135] M. Busl, R. Sánchez, and G. Platero, Phys. Rev. B 81, 121306 (2010).

[136] J. C. Ward, Phys. Rev. 78, 182 (1950).

[137] S. Engelsberg and J. R. Schrieffer, Phys. Rev. 131, 993 (1963).

[138] G. Baym and L. P. Kadanoff, Phys. Rev. 124, 287 (1961).

[139] G. Baym, Phys. Rev. 127, 1391 (1962).

[140] D. A. Ryndyk, A. Donarini, M. Grifoni, and K. Richter, Phys. Rev. B 88, 085404 (2013).

[141] P. Jordan and E. Wigner, Z. Phys. 47, 631 (1928). 